\title{
Toward a High-Resolution Reconstruction of 3D Nerve Fiber Architectures and Crossings in the Brain Using Light Scattering Measurements and Finite-Difference Time-Domain Simulations
}

\author{
Miriam Menzel@, ${ }^{1, *}$ Markus Axer $\odot,{ }^{1}$ Hans De Raedt $\odot,{ }^{2}$ Irene Costantini $\odot,{ }^{3,4}$ Ludovico Silvestri, ${ }^{4}$ \\ Francesco S. Pavone, ${ }^{3,4, \dagger}$ Katrin Amunts $\odot,{ }^{1,5}$ and Kristel Michielsen ${ }^{6}$ \\ ${ }^{1}$ Institute of Neuroscience and Medicine (INM-1), Forschungszentrum Jülich, 52425 Jülich, Germany \\ ${ }^{2}$ Zernike Institute for Advanced Materials, University of Groningen, 9747AG Groningen, Netherlands \\ ${ }^{3}$ National Institute of Optics-Italian National Research Council (INO-CNR), 50125 Firenze, Italy \\ ${ }^{4}$ European Laboratory for Non-Linear Spectroscopy, University of Florence, 50019 Sesto Fiorentino, Italy \\ ${ }^{5}$ Cécile and Oskar Vogt Institute for Brain Research, University Hospital Düsseldorf, \\ University of Düsseldorf, 40204 Düsseldorf, Germany \\ ${ }^{6}$ Jülich Supercomputing Centre, Forschungszentrum Jülich, 52425 Jülich, Germany
}

(Received 31 July 2019; revised manuscript received 28 January 2020; accepted 27 February 2020; published 2 April 2020)

\begin{abstract}
Unraveling the structure and function of the brain requires a detailed knowledge about the neuronal connections, i.e., the spatial architecture of the nerve fibers. One of the most powerful histological methods to reconstruct the three-dimensional nerve fiber pathways is 3D-polarized light imaging (3D-PLI). The technique measures the birefringence of histological brain sections and derives the spatial fiber orientations of whole human brain sections with micrometer resolution. However, the technique yields only a single fiber orientation for each measured tissue voxel even if it is composed of fibers with different orientations, so that in-plane crossing fibers are misinterpreted as out-of-plane fibers. When generating a detailed model of the three-dimensional nerve fiber architecture in the brain, a correct detection and interpretation of nerve fiber crossings is crucial. Here, we show how light scattering in transmission microscopy measurements can be leveraged to identify nerve fiber crossings in 3D-PLI data and demonstrate that measurements of the scattering pattern can resolve the substructure of brain tissue like the crossing angles of the nerve fibers. For this purpose, we develop a simulation framework that permits the study of transmission microscopy measurements - in particular, light scattering — on large-scale complex fiber structures like brain tissue, using finite-difference time-domain (FDTD) simulations and high-performance computing. The simulations are used not only to model and explain experimental observations, but also to develop new analysis methods and measurement techniques. We demonstrate in various experimental studies on brain sections from different species (rodent, monkey, and human) and in FDTD simulations that the polarizationindependent transmitted light intensity (transmittance) decreases significantly (by more than 50\%) with an increasing out-of-plane angle of the nerve fibers and that it is mostly independent of the in-plane crossing angle. Hence, the transmittance can be used to distinguish regions with low fiber density and in-plane crossing fibers from regions with out-of-plane fibers, solving a major problem in 3D-PLI and allowing for a much better reconstruction of the complex nerve fiber architecture in the brain. Finally, we show that light scattering (oblique illumination) in the visible spectrum reveals the underlying structure of brain tissue like the crossing angle of the nerve fibers with micrometer resolution, enabling an even more detailed reconstruction of nerve fiber crossings in the brain and opening up new fields of research.
\end{abstract}

DOI: $10.1103 /$ PhysRevX.10.021002

*m.menzel@fz-juelich.de

${ }^{\dagger}$ Also at Department of Physics, University of Florence, 50019 Sesto Fiorentino, Italy.

Published by the American Physical Society under the terms of the Creative Commons Attribution 4.0 International license. Further distribution of this work must maintain attribution to the author(s) and the published article's title, journal citation, and DOI.
Subject Areas: Biological Physics, Medical Physics, Optics

\section{INTRODUCTION}

The human brain consists of a huge network of nerve fibers: Around 100 billion nerve cells are connected to 10000 other nerve cells on average [1,2]. Understanding the structure and function of the brain remains a key challenge for neuroscience. To figure out how brain function emerges from its structural organization, it is necessary to study the neuronal connections, i.e., the three-dimensional nerve fiber 
architecture of the brain. Developing a detailed network model of the brain, the so-called connectome [3], reveals connected brain regions and helps to identify important nerve fiber connections, which is a prerequisite for brain surgery. Furthermore, the connectivity of the nerve fibers exposes pathological changes in the brain's tissue structure, enabling studies of neurodegenerative diseases like Alzheimer's or Parkinson's disease and the development of new treatments and tools for improved diagnostics.

\section{A. Neuroimaging techniques and their limitations}

Diffusion magnetic resonance imaging (dMRI) is to date the only possibility to study the brain's nerve fiber architecture in living human subjects [4,5]. However, due to motion artifacts and limited scanning times, the resolution of clinical data is limited to the millimeter scale [6,7], so that crossing fiber pathways (i.e., bundles composed of thousands of individual nerve fibers) cannot properly be resolved. Even in postmortem human brains, where dMRI achieves resolutions down to a few hundred micrometers $[8,9]$, fiber pathway tractography algorithms show systematically false-positive fiber pathways due to the lack of detailed knowledge about the fiber crossings [10]. The exact organization principles of nerve fibers in the brain, in particular, fiber crossings, remain a major point of discussion in the MRI community [11-13].

Standard histological methods based on tissue staining $[14,15]$ or histochemistry [16] provide detailed information about the nerve fiber architecture in the cortex (outer surface of the brain) but mostly fail in white matter regions with densely packed nerve fibers. Tracer studies can be used to map individual nerve fibers across long distances but can be applied only to animal brains [17]. To visualize and derive brain tissue properties and organization principles at neuronal scales, light-microscopy techniques are widely used [18,19]. Technological progress and new advances in tissue preparation and labeling have enabled the development of techniques that reveal the 3D nerve fiber architecture in both living and postmortem brains with high resolution [20], such as optical coherence tomography [21-23], microoptical sectioning tomography [24], lightsheet microscopy [25-29], or two-photon fluorescence microscopy (TPFM) [29-33]. However, it is very challenging to apply these techniques to larger tissue samples-in particular, human brains - and to determine fiber orientations in regions with densely packed nerve fibers.

\section{B. 3D-polarized light imaging (3D-PLI)}

The neuroimaging technique 3D-PLI $[34,35]$ is used to study the 3D nerve fiber architectures in whole postmortem human brains with micrometer resolution, bridging several orders of scale. The birefringence of unstained histological brain sections is measured with a polarimeter, thus revealing the spatial orientations of the nerve fibers [36].
The birefringence is mainly caused by the highly ordered molecular structure of the myelin sheaths [37,38] which surround many axons in the white matter of the brain [39]. (In the following, the term nerve fiber is used only for myelinated axons.)

In recent years, 3D-PLI has proven the potential to serve as a validation for fiber tractography algorithms in order to improve the interpretation of clinical dMRI data $[40,41]$. In contrast to dMRI, where thousands of fibers are comprised in one measured tissue voxel, it is only a few tens of fibers in 3D-PLI.

Currently, 3D-PLI is one of the most powerful histological methods for mapping nerve fibers in whole postmortem brains. Because of recent advances, e.g., by using a tiltable specimen stage [42,43], 3D-PLI reliably determines not only the in-plane, but also the out-of-plane orientation of the nerve fibers in most white matter regions. However, 3D-PLI yields only a single fiber orientation for one measured tissue voxel, even if it is composed of crossing fibers with different fiber orientations. As a consequence, brain regions with in-plane crossing fibers are misinterpreted as out-of-plane fibers, i.e., fibers pointing out of the section plane.

\section{Scope of this study}

In this paper, we address this limitation of 3D-PLI and show how crossing nerve fibers and even fiber crossing angles can be determined by exploiting light scattering in brain tissue, providing a major enhancement for reconstructing complex nerve fiber architectures in the brain.

We demonstrate that the transmittance (polarizationindependent transmitted light intensity) of nerve fibers is dominated by isotropic scattering of light and that it contains valuable additional information about the underlying nerve fiber structure. Besides various experimental studies on brains from different species (ranging from rodent and nonhuman primates to humans), we use biophysical modeling and finite-difference time-domain (FDTD) simulations on high-performance computers to explain our experimental observations, create new models for the interpretation of measured data, and develop new imaging methods. Apart from 3D-PLI measurements of brain tissue, the developed simulation framework can be used to model other transmission microscopy techniques and to study light scattering on comparable large-scale complex fiber structures (e.g., muscle fibers, collagen, or artificial fibers).

In this paper, we present two major achievements for an improved reconstruction of the brain's nerve fiber architecture at micrometer resolution.

First, we overcome a major limitation of 3D-PLI, by developing an improved analysis of already measured data that allows for correcting misinterpreted nerve fiber orientations: By taking the transmittance of a measured brain section into account, regions with in-plane crossing fibers 
and regions with low fiber densities can be distinguished from regions with out-of-plane fibers. As the transmittance is determined as part of a standard 3D-PLI measurement, our method can be used to improve the interpretation of already existing data. Moreover, the transmittance can be measured with conventional bright-field transmission microscopes, so that laboratories that are not equipped with special instruments to study 3D fiber architectures can use our findings to extract $3 \mathrm{D}$ information from $2 \mathrm{D}$ transmittance images-without a need to change the experimental setup or to repeat measurements.

Second, we develop a new technique that measures the scattering of light under oblique illumination and reveals the substructure of a measured tissue voxel, in particular, the crossing angle(s) of the nerve fibers, with microscopic resolution for whole brain sections. The technique allows a more detailed reconstruction of nerve fiber crossings in the brain, thus opening up new fields of research.

\section{Outline}

In Sec. II, we present the basic methods of our studies (details are described in Appendix A).

In Sec. III, we present a classification procedure that enables the correction of misinterpreted 3D-PLI signals: We show in both experimental and simulation studies (combining 3D-PLI and TPFM measurements as well as FDTD simulations) that the transmittance of regions with in-plane crossing fibers and low fiber densities differs significantly from regions with out-of-plane fibers and can, therefore, be used to distinguish between these regions. The classification procedure is experimentally validated on known anatomical brain regions.

In Sec. IV, we demonstrate the great potential of scattering measurements to reveal the substructure of brain tissue like the crossing angles of the nerve fibers. First, we show in simulations that the scattering patterns reveal the underlying fiber structure like the in-plane, the out-ofplane, and-most importantly-the crossing angles of the nerve fibers. Then, we show in experimental studies that scattering measurements can indeed be used to determine the correct crossing angle between two nerve fiber bundles. Finally, we demonstrate that the measurement results in known anatomical brain regions correspond very well to the simulated scattering patterns, validating our simulation approach.

\section{MATERIALS AND METHODS}

\section{A. Preparation of brain sections}

The measurements are performed on healthy brains from mice, rats, vervet monkeys, a hooded seal, and humans. All brains are obtained directly after death in accordance with legal and ethical requirements. The brains are deeply frozen, cut into sections of $60 \mu \mathrm{m}$ thickness, embedded in a solution of $20 \%$ glycerin, cover slipped, and measured
1-2 days afterward. A detailed description of the brain preparation can be found in Appendix A 1.

\section{B. 3D-PLI measurement}

The 3D-PLI measurements are performed using a polarizing microscope with a numerical aperture of 0.15 and an object-space resolution of about $1.33 \mu \mathrm{m}$ per pixel [34,35]. The microscope consists of an LED light source with $550 \mathrm{~nm}$ wavelength, a rotating linear polarizer, a specimen stage containing the brain section, a circular polarization analyzer, and a camera which records the transmitted light intensity for different rotation angles $\left\{0^{\circ}, 10^{\circ}, \ldots, 170^{\circ}\right\}$ of the polarizer. More information about the 3D-PLI measurement can be found in Appendix A 2.

The amplitude of the measured intensity signal (retardation $|\sin \delta|$ ) is related to the birefringence of the brain section and serves as a measure of the out-of-plane inclination angle $\alpha$ of the nerve fibers, using $\delta \propto \cos ^{2} \alpha$ [36]. As the out-of-plane inclination is considered independently from the in-plane orientation of the fibers, all inclination angles are given as absolute values without sign $\left(\alpha \in\left[0^{\circ}, 90^{\circ}\right]\right)$.

The transmittance is computed from the same signal without additional measurements by averaging the measured light intensities over all rotation angles. To consider only effects caused by the brain tissue, the resulting transmittance values are normalized for each image pixel by the average transmitted light intensity without a sample (normalized transmittance $I_{T, N}$ ).

\section{TPFM measurement}

The TPFM measurements are performed with a custommade two-photon fluorescence microscope with a wavelength of $800 \mathrm{~nm}[29,44]$. The microscope achieves a resolution of $0.244 \times 0.244 \times 1 \mu \mathrm{m}^{3}$ and allows in-depth scans of the sample (see Appendix A 3 for more details).

Brain tissue exhibits weak intrinsic autofluorescence which slightly differs between different tissue components. Therefore, TPFM measurements of brain tissue can be used to manually separate nerve fiber bundles from surrounding tissue.

\section{Light scattering measurement}

The scattering measurements are performed by placing a mask with a hole on top of an LED array (525 nm wavelength) so that the center of the sample is illuminated under a polar angle between $47^{\circ}$ and $49^{\circ}$ with respect to the section plane normal (see Fig. 10 in Appendix A 5). During the measurement, the mask is rotated in equidistant steps around the center of the sample, and an image is recorded for each rotation angle. The resolution in object space is about $6.5 \mu \mathrm{m}$. In Appendix A 5, the measurement setup is described in more detail. 


\section{E. FDTD simulation}

The FDTD simulations enable studies of light scattering in brain tissue with microscopic detail. The algorithm discretizes time and space, models the propagation of the light wave by approximating the spatial and temporal derivatives in Maxwell's curl equations by second-order central differences, and numerically computes the electromagnetic field components in space and time [45-49]. As the mesh size of the spatial discretization needs to be much smaller than the wavelength (at most $25 \mathrm{~nm}$ ), simulations of tissue samples with dimensions of several micrometers are computationally very intense. Therefore, a simplified simulation model for the brain tissue and the optics of the imaging system is developed, enabling studies of larger fibrous tissue samples.

To examine the transmittance and scattering of light, the 3D-PLI measurement is simulated for various artificial nerve fiber configurations. The fiber configurations consist of about 700 fibers with uniformly distributed diameters between 1.0 and $1.6 \mu \mathrm{m}$ and different fiber orientations. All fibers are generated in a volume of $30 \times 30 \times 30 \mu \mathrm{m}^{3}$ without intersections. (The generation of the fiber configurations is described in Appendix $\mathrm{C}$ in more detail.) Each fiber is represented by a simplified nerve fiber model, consisting of an inner axon with a constant radius and a surrounding myelin sheath with two layers, defined by different refractive indices (see Appendix D).

The propagation of the light wave through the tissue sample (artificial fiber configuration) is computed by a conditionally stable FDTD algorithm (see Appendix E). The resulting electric field components are processed with analytical methods taking all optical components of the polarizing microscope into account, including the objective lens (with numerical aperture NA $=0.15$ ) and the camera detector (see Appendix G).

All simulation studies in the subsequent sections are performed for normally incident light with $550 \mathrm{~nm}$ wavelength and for the simulation parameters listed in Appendix F. One simulation run (volume of $30 \times 30 \times$ $30 \mu \mathrm{m}^{3}$, mesh size of $25 \mathrm{~nm}$ ) consumes about 8000 core hours on the supercomputer JUQUEEN using an MPI (message passing interface) Cartesian grid of $16 \times 16 \times 16$, allowing for many simulation runs with different parameters. The accuracy of the simulation results is discussed in Appendix H.

\section{CORRECTING MISINTERPRETATIONS IN 3D-PLI}

Figure 1 shows the reconstructed nerve fiber orientations of a vervet monkey brain section obtained from a 3D-PLI measurement (the three-dimensional nerve fiber orientations are encoded in different colors; see the color bubble in the upper right corner). In a standard 3D-PLI analysis, the out-of-plane fiber orientation angle $\alpha$ is computed from the



FIG. 1. Fiber orientation map of a coronal vervet monkey brain section, obtained from a 3D-PLI measurement with $1.33 \mu \mathrm{m}$ pixel size. The enlarged area highlights three brain regions that all yield low birefringence signals: (i) gray matter with low fiber density, (ii) crossing nerve fibers (corona radiata), and (iii) steep out-of-plane fibers (fornix).

measured birefringence signal $|\sin \delta|$, assuming $\delta \propto \cos ^{2} \alpha$ (valid for dense parallel nerve fibers [36]). Thus, regions with a low birefringence signal $(\delta \ll 1)$ are interpreted as steep (out-of-plane) fibers $(\alpha \gg 1)$. The enlarged area illustrates that there exist three different types of brain tissue that all yield low birefringence signals: (i) gray matter regions with low fiber density which contain less birefringent tissue components and therefore yield a low birefringence signal, (ii) regions with crossing fibers in which the birefringence signals cancel out, and (iii) regions with actual out-of-plane fibers.

In this section, we correct for the misinterpretations in 3D-PLI: We show that the transmittance of regions with low fiber density and regions with in-plane crossing fibers differs significantly from regions with out-of-plane fibers and can, therefore, be used to distinguish them. The inclination of the out-of-plane fibers can then be derived using standard 3D-PLI analysis.

The transmittance is a measure of how much the light is attenuated when it passes through the brain tissue; i.e., it depends on tissue absorption as well as scattering of light. As the absorption coefficient of brain matter is small (less than $0.1 \mathrm{~mm}^{-1}[50,51]$ ), the measured transmittance is expected to be mainly influenced by scattering. To study such complex light-tissue interactions at the microscopic level and explain the experimental observations, we employ FDTD simulations to compute the propagation of the light wave through the brain tissue sample [45-49]. 
In regions with low fiber density (gray matter), it is already known that the transmittance is notably higher than in regions with densely packed nerve fibers (white matter) due to the high attenuation coefficient of white matter [50,51]. In Sec. III A, we show that the transmittance decreases with an increasing out-of-plane inclination angle of the enclosed nerve fibers; i.e., the transmittance depends on the orientation of the nerve fibers with respect to the light beam. In Sec. III B, we show that the transmittance does not depend on the crossing angle of in-plane nerve fibers. Finally, in Sec. III C, we demonstrate that regions with inplane crossing fibers and regions with low fiber density can be distinguished from regions with out-of-plane fibers by a combined analysis of the transmittance and the strength of the measured birefringence signal.

\section{A. Transmittance of inclined nerve fibers}

First, we show in various experimental studies (Sec. III A 1) that the transmittance of mostly parallel, densely packed nerve fibers decreases significantly (by more than 50\%) with an increasing out-of-plane inclination angle of the fibers. In the subsequent simulation studies (Sec. III A 2), we demonstrate that this decrease is mainly caused by isotropic light scattering and by the finite numerical aperture of the imaging system.

\section{Experimental studies}

The transmittance of a brain section depends on many factors which differ from tissue to tissue, such as the degree of myelination, the density of the nerve fibers, the type of brain tissue, the species, the preparation of the tissue, or the exact tissue composition.

Myelination and fiber density are less relevant in this section, because we consider only white matter regions with densely packed, myelinated nerve fibers. In order to make general statements about the inclination dependence of the transmittance-independently from intersubject differences and variations in tissue preparation or composition-we combine studies on different species (rodent, monkey, and human), subjects, and brain sections. To access the fiber inclination, we use both 3D-PLI and TPFM measurements as well as analyses of different anatomical planes and 3D reconstruction. For reasons of clarity, we here provide only a summary of the most important results. The whole study is described in Appendix B.

To figure out how the transmittance of a brain region depends on the orientation of the nerve fibers with respect to the section plane, we investigate sections from brains that are cut along mutually orthogonal anatomical planes: One brain is cut along the coronal plane (dividing the brain into back and front), and the other brain is cut along the sagittal plane (dividing the brain into left and right). As the sagittal plane is oriented perpendicular to the coronal plane, the transmittance of the same anatomical brain region can be evaluated for flat (in-plane) nerve fibers in one section (a)

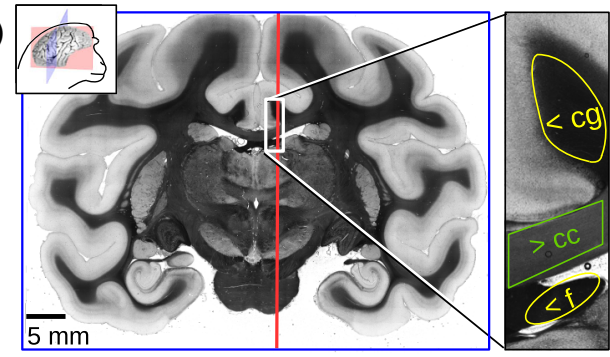

(b)

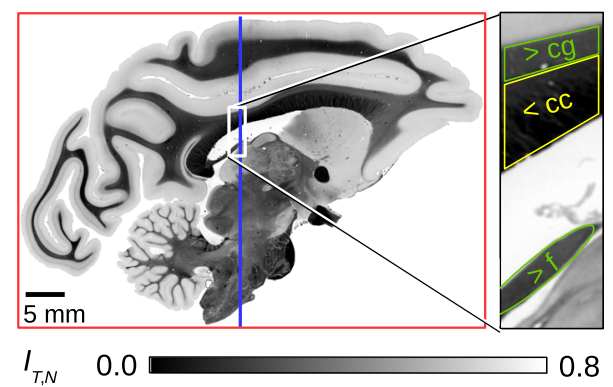

FIG. 2. Normalized transmittance images $I_{T, N}$ of a coronal (a) and a sagittal (b) vervet brain section, obtained from a 3D-PLI measurement with $1.33 \mu \mathrm{m}$ pixel size (the fiber orientation map in Fig. 1 is obtained from the same coronal brain section). For reference, the coronal (sagittal) section plane is indicated by a vertical blue (red) line in the respective other brain section. The enlarged areas on the right show anatomical brain regions with predominantly in-plane (out-of-plane) nerve fibers in green (yellow). Within each section, in-plane nerve fibers have larger transmittance values than out-of-plane fibers. $\mathrm{cg}=$ cingulum, $\mathrm{cc}=$ corpus callosum, and $\mathrm{f}=$ fornix .

plane and for steep (out-of-plane) nerve fibers in the other section plane.

Figure 2 shows the transmittance images for a coronal and a sagittal section of a vervet monkey brain. The approximate orientation of the nerve fibers is known from vervet brain atlases [52-54]: In the coronal section plane, fibers in the cingulum (cg) and the fornix (f) are mostly oriented out of plane, while fibers in the corpus callosum (cc) are mostly oriented in plane. In the sagittal section plane, it is exactly the other way around.

In each brain section, regions with steep nerve fibers (marked in yellow) have more than 50\% lower transmittance values than regions with flat nerve fibers (marked in green). As expected, images obtained from conventional bright-field transmission microscopy with unpolarized light show similar effects. The exact transmittance values and evaluation of other anatomical regions-including rat and human brain samples - can be found in Appendix B 1.

As always, when the same anatomical brain region is investigated in different section planes, it is reasonable to assume that differences in the tissue structure (myelination, fiber density, and tissue composition) are small. Differences in tissue preparation and interspecimen differences are addressed by only comparing transmittance values within one brain section. 
In a further study (see Appendix B 2), we examine the transmittance of several consecutive brain sections that are registered onto each other. While there are no interspecimen differences, the 3D-reconstructed volume of transmittance images shows that the average transmittance differs between brain sections, most likely due to minor differences in tissue preparation. Nevertheless, our observation that steep nerve fibers appear much darker than inplane nerve fibers (within each brain section) is consistent across hundreds of consecutive brain sections and large anatomical structures.

In regions with distinct nerve fiber bundles, like the caudate putamen of rodent brains, the fiber inclination can be estimated by manually evaluating the course of the fiber bundles in different section planes. We study the transmittance contrast between nerve fibers and surrounding gray matter in mutually orthogonal section planes of the caudate putamen (see Appendix B 3). As gray matter can be considered to be mostly independent of the section plane, it is used as a reference. We find once again that steep fiber bundles (with respect to the section plane) show lower transmittance values than flat fiber bundles.

With TPFM measurements, we finally reveal the substructure of the caudate putamen, determine the inclination angles of individual fiber bundles, and compare them to the corresponding transmittance values of the measured brain section [see Fig. 3(a)]. The resulting scatter plot shows a clear tendency toward a decrease in transmittance with an increasing fiber inclination angle. As expected, the transmittance for regions with maximum fiber density (blue) is lower than for regions with reduced fiber density (orange), but it decreases even further with an increasing inclination angle. This result shows that low transmittance values in regions with densely packed nerve fibers are mainly caused by inclined nerve fibers. (Appendix B 4 provides a more detailed evaluation.)

All our experimental studies show that the transmittance of brain tissue decreases steadily and significantly (by more than 50\%) with an increasing out-of-plane inclination angle of the enclosed nerve fibers.

\section{Simulation studies}

Although the experimental studies clearly demonstrate that the transmittance decreases with an increasing inclination angle of the nerve fibers, they do not provide enough information to explain this effect in detail. To develop a model and better understand the observed transmittance effect, we perform numerical simulations on artificial nerve fiber configurations with different inclination angles. This simulation has the advantage that the exact underlying fiber structure and, thus, the inclination angles of the nerve fibers are known-also in bulk tissue with densely packed fibers.

We generate an artificial bundle of densely grown fibers [see Fig. 3(b)(i) and Appendix C 1] for different inclination angles $\alpha=\left\{0^{\circ}, 10^{\circ}, \ldots, 90^{\circ}\right\}$ and compute the
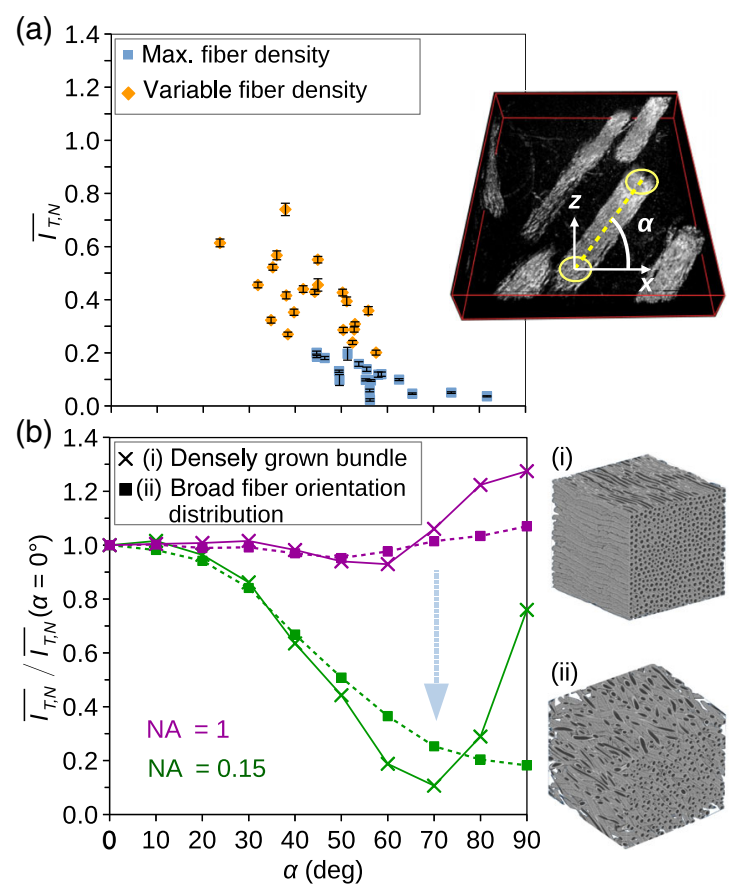

FIG. 3. Transmittance of inclined fiber bundles. (a) Mean normalized transmittance values $\overline{I_{T, N}}$ plotted against the nerve fiber inclination angles $\alpha$ determined, respectively, from 3D-PLI and TPFM measurements of nerve fiber bundles in a mouse brain section (see Fig. S2 in Supplemental Material [55]). The values in blue belong to regions with similar (maximum) fiber density, and the values in orange belong to regions with variable fiber density in which the transmittance might be overestimated. The error bars indicate the standard error of the mean for the evaluated transmittance values. (b) Simulated transmittance curves (mean transmittance $\overline{I_{T, N}}$ vs inclination $\alpha$ ) for a bundle of densely grown fibers (i) and a bundle with broad fiber orientation distribution (ii) for $\mathrm{NA}=1$ (purple curves) and NA $=0.15$ (green curves). The transmittance curves are normalized by the mean transmittance values of the horizontal bundles, respectively. The simulations are performed with the parameters specified in Appendix F, using normally incident light with $550 \mathrm{~nm}$ wavelength. Both experimental and simulated data show that the transmittance decreases with an increasing fiber inclination (for $\mathrm{NA}=0.15$ ).

transmittance from a simulated 3D-PLI measurement (see Sec. IIE). To study the effect of the finite numerical aperture of the imaging system on the measured transmittance values, we simulate the imaging system without an aperture $(\mathrm{NA}=1)$ considering light scattered under all angles and with an aperture $(\mathrm{NA}=0.15)$ considering only light scattered under angles $<8.6^{\circ}$. The resulting transmittance images and scattering patterns can be found in Fig. S3 in Supplemental Material [55].

Figure 3(b) shows the resulting transmittance curves (mean values of the simulated transmittance images plotted against the inclination angles of the fiber bundle) for $\mathrm{NA}=1$ (purple curves) and $\mathrm{NA}=0.15$ (green curves). The solid curves are obtained from the bundle of densely grown fibers (i) which has similar fiber orientations (the 
mode angle difference between the local fiber orientation vectors and the predominant orientation of the fiber bundle is less than $10^{\circ}$; see Appendix C 1). The dashed curves are obtained for a bundle with broad fiber orientation distribution (ii) which contains many different fiber orientations (the mode angle difference is about $25^{\circ}$; see Appendix C 2). To enable a better comparison between the different curves, all curves are normalized by the mean transmittance value of the horizontal bundle $\left(\alpha=0^{\circ}\right)$, respectively.

For NA $=1$, the transmittance for steep fibers $\left(\alpha>45^{\circ}\right)$ is similar to or even slightly larger than the transmittance for flat fibers $\left(\alpha<45^{\circ}\right)$. For NA $=0.15$, the transmittance decreases significantly between $\alpha=30^{\circ}$ and $\alpha=70^{\circ}$. Hence, the observed decrease in transmittance is caused by the finite numerical aperture of the imaging system: For steep fibers, the light is scattered almost uniformly in all possible directions [see Fig. S3(c) in Supplemental Material [55] for $\alpha=70^{\circ}$ ] so that the detected transmitted light intensity becomes minimal. In simulation studies with polarized light, we could show that the decrease in transmittance is independent of the direction of polarization [see Fig. 18(a) in Appendix H], which suggests that the decrease is caused by isotropic (not by anisotropic) scattering of light. For vertical fibers $\left(\alpha=90^{\circ}\right)$, the transmittance increases again. In real brain tissue, however, it is rather unlikely that a tissue voxel is completely filled with vertical fibers over the whole section thickness of $60 \mu \mathrm{m}$, so this behavior is not expected to be commonly observed in the measurement.

The transmittance for the bundle with broad fiber orientation distribution decreases monotonically with an increasing fiber inclination angle and becomes minimal for vertical fibers (the transmittance for vertical fibers is more than $80 \%$ less than for horizontal fibers). Because of the broad fiber orientation distribution, the vertical bundle contains many fibers with inclinations between $60^{\circ}$ and $70^{\circ}$, which explains why the minimum transmittance is shifted to larger inclination angles.

Especially for the bundle with broad fiber orientation distribution, the simulated transmittance curves [Fig. 3(b)] show a similar behavior as the measured transmittance values in the scatter plot [Fig. 3(a)].

\section{B. Transmittance of crossing nerve fibers}

In the previous section, we show that the transmittance of brain tissue depends on the out-of-plane angle of the nerve fibers. In this section, we examine how the transmittance depends on the in-plane crossing angle of the fibers. Both experimental studies (Sec. III B 1) and simulation studies (Sec. III B 2) reveal that the transmittance is mostly independent of the crossing angle.

\section{Experimental studies}

To study the transmittance of in-plane crossing fibers, we consider the optic chiasm of a hooded seal [56] — a region where the two optic nerves of the brain cross each other [see Fig. 4(c)]. The section plane is chosen such that it contains mostly in-plane nerve fibers with a broad distribution of crossing angles.

Figure 4(b) shows the transmittance and retardation images for the middle section of the chiasm. As expected from the broad distribution of crossing angles [cf. Fig. 4(c)], the retardation values in the region with crossing fibers (region $B$ in orange) are broadly distributed, because the birefringence signals of crossing fibers partly cancel out

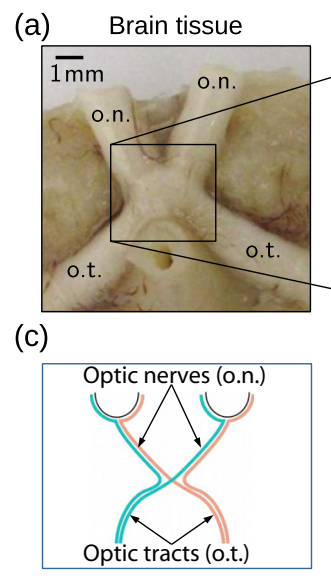

(b)
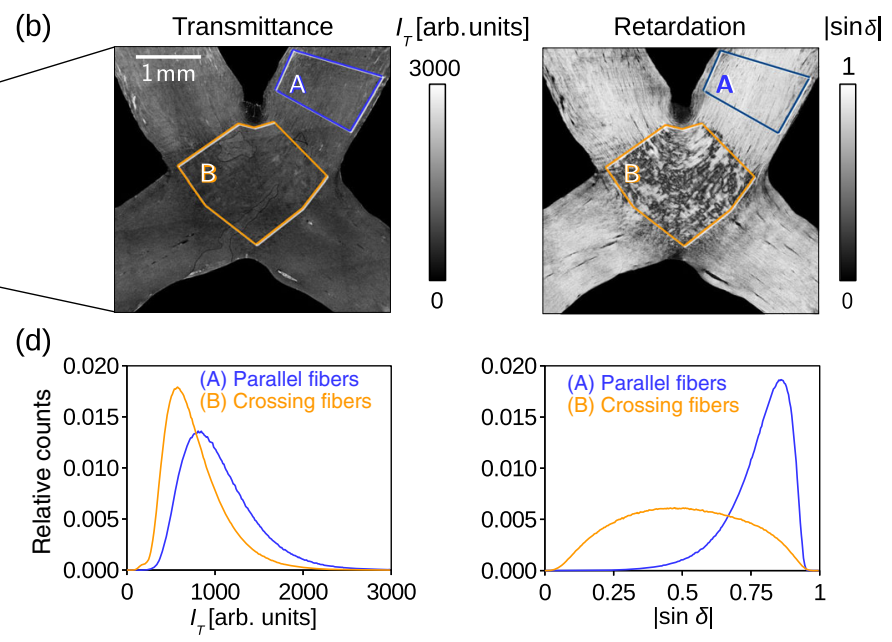

FIG. 4. Crossing nerve fibers in the optic chiasm of a hooded seal: (a) brain tissue before sectioning, (b) unnormalized transmittance and retardation images of the middle brain section obtained from 3D-PLI measurements with $1.33 \mu$ m pixel size, (c) schematic drawing of the optic chiasm consisting of optic tracts (o.t.) and optic nerves (o.n.), (d) normalized histograms of the transmittance image $\left(I_{T}\right)$ and retardation image $\left(|\sin \delta|\right.$ ) for a region with mostly parallel fibers (blue) and a region with nearly $90^{\circ}$-crossing fibers (orange). Unlike the retardation, the transmittance does not depend on the crossing angles of the nerve fibers, only on the tissue density. More information about the sample can be found in Dohmen et al. [56] [(a) and (c) are adapted from Figs. 1 and 5B0 in [56] copyright 2015, with permission from Elsevier]. 
(depending on the crossing angle). In contrast to the retardation, the transmittance values in the crossing region show a similar distribution as in a region with mostly parallel fibers (region $A$ in blue); see the histograms in Fig. 4(d). The peak transmittance value of region $B$ is slightly lower than in region $A$, because the number of fibers in the crossing region (two crossing bundles) is larger than in the region with parallel fibers (one bundle). Thus, the transmittance depends on the tissue density but not on various crossing angles between the nerve fibers.

\section{Simulation studies}

Although the experimental results show that the transmittance is mostly independent from various crossing angles, the exact substructure and crossing angles of the fibers are unknown. To study the effect in more detail, we simulate the transmittance of horizontal (in-plane) crossing fibers for different crossing angles and compare the results to the transmittance of steep (out-of-plane) fibers. The horizontal crossing fibers are generated as separate and interwoven fiber bundles [see Figs. 5(a) and 5(b) and Appendix C2] with different crossing angles $\chi=\left\{0^{\circ}\right.$, $\left.15^{\circ}, \ldots, 90^{\circ}\right\}$. In addition, we study the transmittance for three mutually orthogonal, interwoven fiber bundles [see Fig. 5(c) and Appendix C 2].

Figure 5(d) shows the mean transmittance values of the different fiber bundles for NA $=0.15$ plotted against the crossing angle $\chi$ (in the case of horizontal crossing fibers). (a)
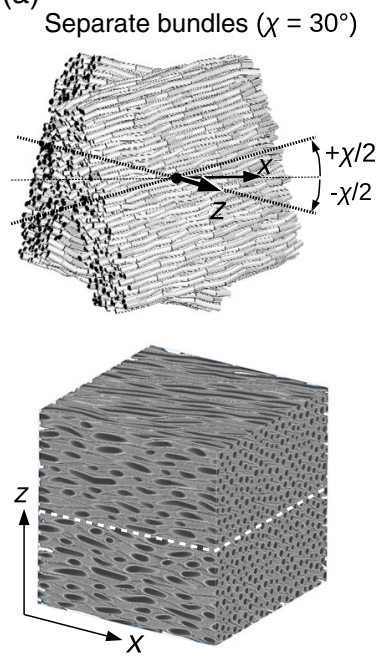

(b)


(d)

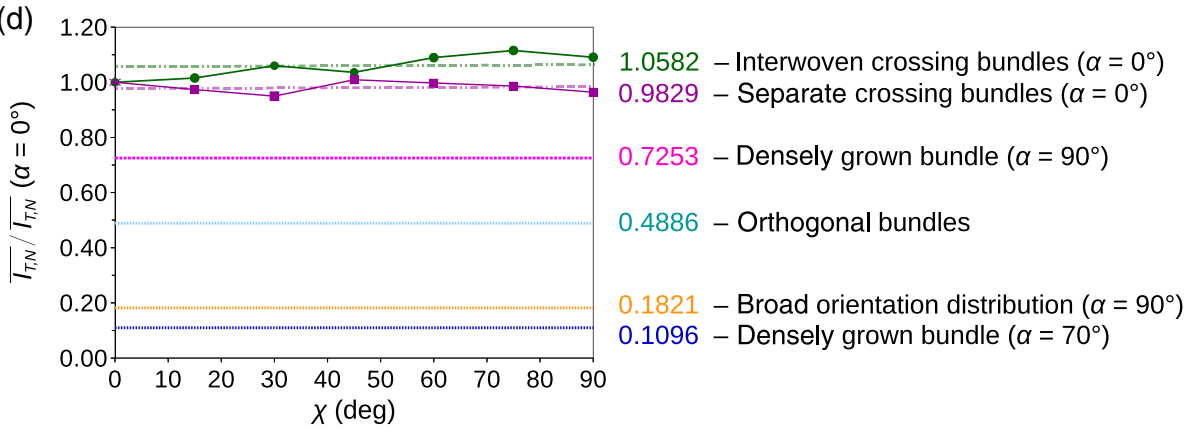

(c)
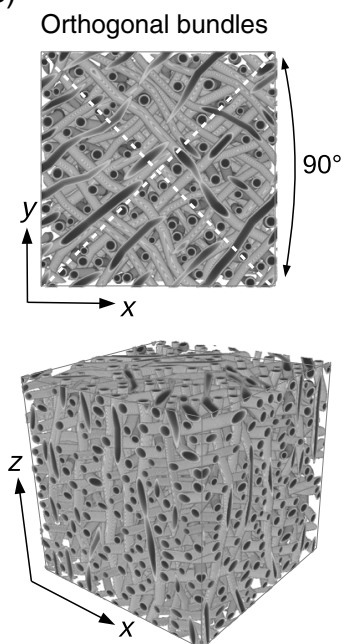

FIG. 5. Simulated transmittance of crossing fibers. (a),(b) Separate and interwoven fiber bundles with crossing angle $\chi$ : The upper figures show the generated bundles before cropping. The lower figures show the bundles after being cropped to a volume of $30 \times 30 \times 30 \mu \mathrm{m}^{3}$. The white dotted line indicates the border between the upper and the lower bundle of the separate crossing fibers. (c) Three mutually orthogonal, interwoven fiber bundles cropped to a volume of $30 \times 30 \times 30 \mu \mathrm{m}^{3}$. The white dotted lines indicate the main directions of the two horizontal fiber bundles in the $x y$ plane, and the third fiber bundle is oriented in the $z$ direction. All fiber configurations are generated from 700 fibers with diameters between 1.0 and $1.6 \mu \mathrm{m}$. (d) Mean transmittance values for different crossing angles $\chi$ shown for in-plane crossing (solid curves) and out-of-plane fiber configurations (densely dotted curves). For better comparison, the values are divided by the mean transmittance value of the corresponding horizontal fiber bundle for $\chi=0^{\circ}$. The mean normalized transmittance values $\overline{I_{T, N}}$ are computed from a simulated 3D-PLI measurement (with numerical aperture NA $=0.15$ ). The simulations are performed with the parameters specified in Appendix F, using normally incident light with $550 \mathrm{~nm}$ wavelength. Apart from the fiber configurations shown in this figure, the mean transmittance values are also displayed for the bundle of densely grown fibers [see Fig. 3(b)(i)] for $\alpha=70^{\circ}$ and $90^{\circ}$ and for a vertical fiber bundle with broad fiber orientation distribution [see Fig. 3(b)(ii)]. The mean transmittance is mostly independent of the crossing angle and larger than the mean transmittance of out-of-plane fibers. 
For better comparison, the values are divided by the mean transmittance value of the corresponding horizontal fiber bundle (for $\chi=0^{\circ}$ ), respectively. The solid curves belong to the horizontal crossing fibers (separate and interwoven bundles), and the densely dotted lines below belong to fiber constellations that contain vertical or steep fibers: the bundle of densely grown fibers for $\alpha=90^{\circ}$ and $70^{\circ}$ [cf. Fig. 3(b)(i)], the mutually orthogonal fiber bundles [cf. Fig. 5(c)], and the bundle with broad fiber orientation distribution for $\alpha=90^{\circ}$ [cf. Fig. 3(b)(ii)].

The transmittance curves of horizontal crossing fibers are similar for separate and interwoven fiber bundles. The mean transmittance of the separate crossing fibers corresponds more or less to the mean transmittance of the horizontal fiber bundle for $\chi=0^{\circ}$. The transmittance values of the interwoven crossing fibers slightly increase with an increasing crossing angle (by maximum $11 \%$ ) and are up to $13 \%$ larger than those for the separate fiber bundles.

For the vertical bundle of densely grown fibers, which is unlikely to occur in real brain tissue and shown only as a limiting case, the mean transmittance value is already more than 26\% less than for the horizontal crossing fibers. For interwoven crossing fibers, the transmittance value is reduced by more than one-half when the horizontal crossing fibers are combined with a vertical fiber bundle (orthogonal bundles). For the vertical bundle with broad fiber orientation distribution and the steep bundle of densely grown fibers (with $\alpha=70^{\circ}$ ), the difference between the transmittance values is especially large: The transmittance is about $80 \%-90 \%$ less than for the horizontal crossing fibers.

Our studies show that the transmittance for in-plane fibers is mostly independent of the crossing angle between the bundles and much larger than the transmittance for outof-plane fibers. This finding suggests that the transmittance values can be used to distinguish in-plane crossing fibers from out-of-plane fibers in 3D-PLI measurements and to detect out-of-plane fibers within fiber crossings.

\section{Classification of misinterpreted 3D-PLI signals}

Based on the results from the previous sections, we develop a classification procedure that allows for correcting misinterpreted 3D-PLI signals, i.e., identifying regions with low fiber density and in-plane crossing fibers, which are misinterpreted as out-of-plane fibers due to their low birefringence signals: By using a combined analysis of transmittance and retardation images, it is possible to classify regions with small birefringence signals into regions with low fiber density (higher transmittance than in-plane parallel fibers), in-plane crossing fibers (similar transmittance as in-plane parallel fibers; see Sec. III B), and out-of-plane fibers (lower transmittance than in-plane parallel fibers; see Sec. III A). To demonstrate the functionality of the classification procedure, we consider a coronal section (right occipital lobe) of a vervet monkey brain (see Fig. 6).

Since the transmittance depends on the fiber density, the region with maximum fiber density is used as a reference: The retardance $\delta$ of brain tissue becomes maximal for a region with in-plane fibers $(\alpha=0)$ and maximum thickness $d$ of birefringent tissue components $\left(\delta \propto d \Delta n \cos ^{2} \alpha\right.$, where $\Delta n$ is the birefringence of the tissue [34]). Assuming that a brain section contains a large variety of nerve fiber configurations, the region with maximum retardation signal $|\sin \delta|_{\max }$ (orange ellipse in Fig. 6) is therefore expected to contain mostly in-plane parallel fibers $\left(\alpha \approx 0^{\circ}\right)$ with a high fiber density (maximum $d \Delta n$ ). Regions with even lower transmittance values are accordingly expected to contain steep (out-of-plane) fibers which increase the scattering and, thus, the attenuation of light.

By comparing the normalized transmittance values $\left(I_{T, N}\right)$ of regions with small retardation values to the transmittance of the region with maximum retardation $\left[I_{\text {ref }} \equiv I_{T, N}\left(|\sin \delta|_{\max }\right)\right]$, the regions can be classified into three categories (see Fig. 6):
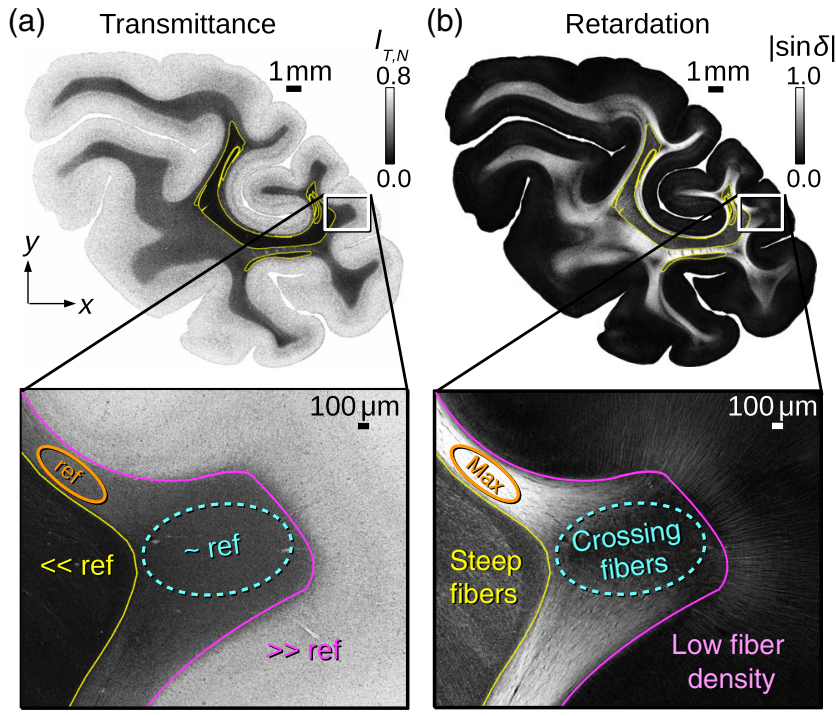

FIG. 6. Combined analysis of transmittance and retardation images allowing the classification of brain regions with low birefringence signals in 3D-PLI measurements. The figure shows the normalized transmittance image $\left(I_{T, N}\right)$ and the retardation image $(|\sin \delta|)$ of a coronal section through the right hemisphere (occipital lobe) of a vervet monkey brain [cf. Fig. 13(a) in Appendix B 2] obtained from a 3D-PLI measurement with $1.33 \mu \mathrm{m}$ pixel size. The transmittance in the region with maximum retardation (orange ellipse) is used as a reference value: Regions with small retardation values and notably lower transmittance values (regions surrounded by a yellow line) are expected to contain steep (out-of-plane) fibers. Regions with small retardation values and similar transmittance values (cyan) are expected to contain flat (in-plane) crossing fibers. Regions with small retardation values and larger transmittance values (purple) belong to regions with low fiber density, i.e., regions with a large amount of unmyelinated axons or surrounding tissue. 
(i) $I_{T, N} \ll I_{\text {ref }}$ - - Regions with notably lower transmittance values are expected to contain steep (out-ofplane) fibers (yellow);

(ii) $I_{T, N} \sim I_{\text {ref }}$ - - Regions with similar transmittance values are expected to contain flat (in-plane) crossing fibers (cyan);

(iii) $I_{T, N} \gg I_{\text {ref }}$. - Regions with notably larger transmittance values are expected to have a lower fiber density (purple).

For regions with slightly lower or larger transmittance values, an unambiguous classification is not possible. Provided that the region with maximum retardation has the largest tissue absorption, lower transmittance values can be caused only by out-of-plane fibers. Similar transmittance values, however, could also be caused by a small number of out-of-plane fibers, and larger transmittance values could be caused by a small number of in-plane crossing fibers (or a smaller number of out-of-plane fibers). A classification by means of retardation and transmittance values can, therefore, serve only as an indication of the underlying fiber configuration and should always be considered in addition to individual tissue characteristics. As the transmittance depends on the tissue preparation, the combined analysis of transmittance and retardation should be performed only sectionwise. Brain atlases and 3D-reconstructed images (see Fig. 13 in Appendix B 2) validate the classification of regions in Fig. 6.

After identifying the regions with low fiber density and in-plane crossing fibers which are misinterpreted as steep fibers, the determined out-of-plane inclination angles of the remaining regions with steep fibers can be considered as reliable.

\section{SCATTERING MEASUREMENTS OF BRAIN TISSUE}

The classification procedure presented in the previous section allows an automated identification of nerve fiber crossings in 3D-PLI measurements. However, it is not possible to determine the exact substructure of the tissue, e.g., the crossing angles of the nerve fibers, without considerable manual effort. In the following, we show the potential of scattering measurements to reveal the substructure of measured tissue voxels for a whole brain section with micrometer resolution.

\section{A. Scattering patterns reveal tissue substructure}

Figure 7 shows the simulated scattering patterns for the nerve fiber configurations investigated in Sec. III: (a) densely grown fiber bundle with different inclination angles [cf. Fig. 3(b)(i)] and (b) separate and interwoven fiber bundles with different crossing angles [cf. Figs. 5(a) and 5(b)]. The scattering patterns and transmittance images for all simulated inclination angles $\left(0^{\circ}, 10^{\circ}, \ldots, 90^{\circ}\right)$ can be found in Fig. S3 in Supplemental Material [55]. The scattering patterns show the intensity per wave vector angle $\theta_{k}$ of light transmitted through the sample. The (a)

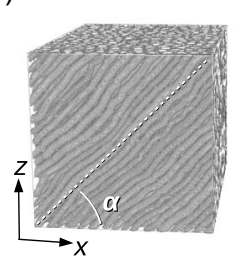

(b)
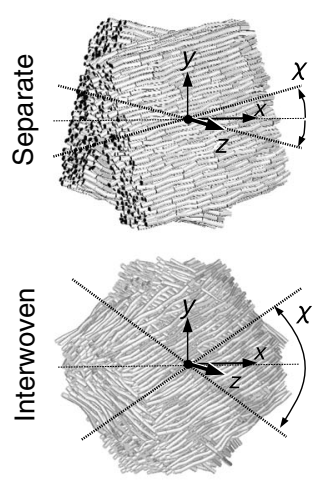
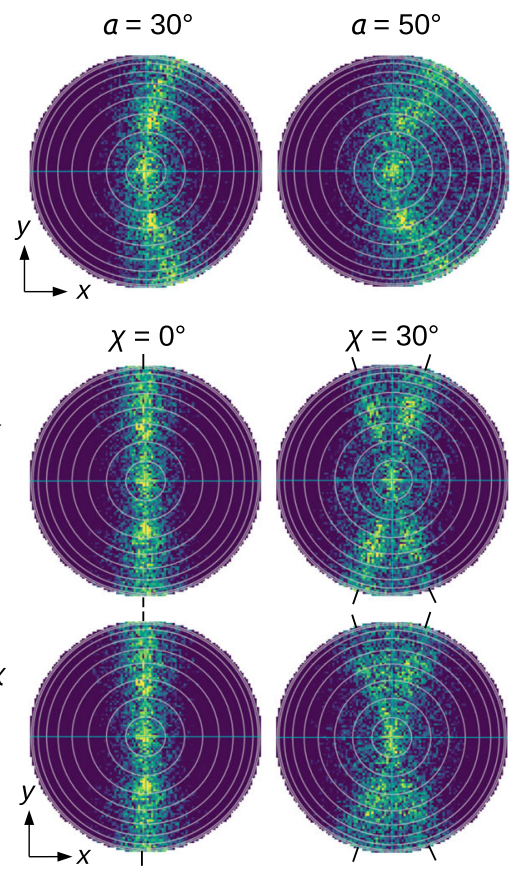
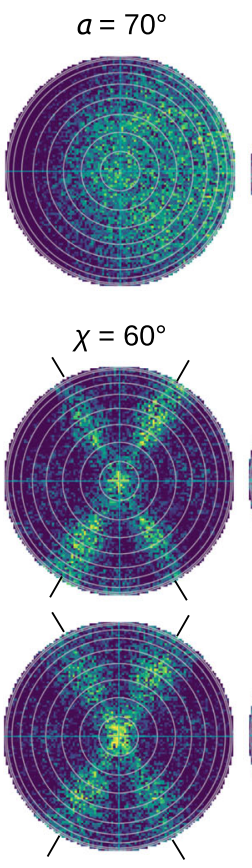
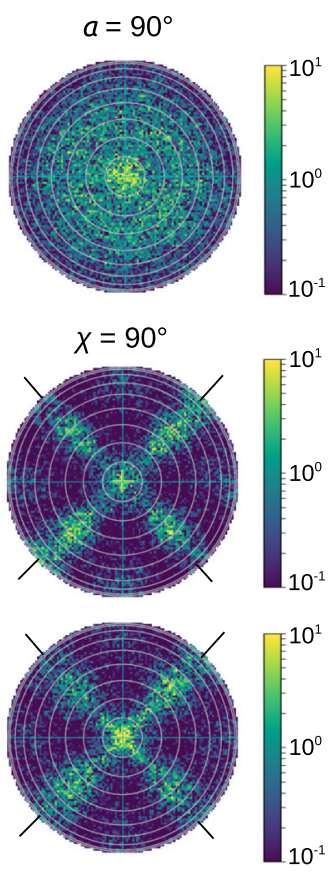

FIG. 7. Simulated scattering patterns for different artificial nerve fiber constellations: (a) densely grown fiber bundle [cf. Fig. 3(b)(i)] with different inclination angles $\alpha$ and (b) in-plane crossing fibers [separate and interwoven bundles; cf. Figs. 5(a) and 5(b)] with different crossing angles $\chi$. The scattering patterns show the underlying substructure, e.g., the crossing angle of the fibers (indicated by the black lines around the patterns). 
white circles represent steps of $\Delta \theta_{k}=10^{\circ}$, from $0^{\circ}$ (center) to $90^{\circ}$ (outer circle).

For in-plane fibers $\left(\alpha<30^{\circ}\right)$, the light is mostly scattered under angles perpendicular to the principal axis of the fiber bundle (i.e., along the $y$ axis). For intermediate inclination angles, the light is scattered more and more in the direction of the fibers (i.e., in the positive $x$ direction). The minimum angular distance between the maxima decreases with an increasing inclination angle. For an inclination angle of $70^{\circ}$, the light is broadly scattered in the direction of the fibers (positive $x$ axis); see Fig. 7(a). For an inclination angle of $90^{\circ}$, the light is uniformly scattered in all directions.

The simulated scattering patterns of separate and interwoven crossing fiber bundles look similar for all crossing angles [see Fig. 7(b)]. The underlying fiber configuration, i.e., the crossing angle of the fiber bundles, is clearly visible in all scattering patterns.

The simulations suggest that a measurement of the scattering pattern provides valuable information about the tissue substructure, in particular, the crossing angle of the nerve fibers. In the simulation, the light falls vertically onto the sample and is scattered in different directions behind the sample; the computed scattering pattern shows the intensity of the scattered light for different scattering angles. For the scattering measurement, we take advantage of the fact that the light path is reversible: Instead of measuring the scattering pattern, we illuminate the sample from different angles (oblique illumination) and record the light that falls vertically onto the camera. To extract the major features of the scattering pattern like the direction, inclination, and crossing angle of the fibers, it is enough to consider only one angle of scattering, i.e., one outer circle in the simulated scattering pattern [cf. white dashed circles in Fig. 9(b)]. We therefore perform our measurements with a fixed polar angle of illumination with respect to the section plane normal (around $47^{\circ}-49^{\circ}$ ) and rotate the point of illumination (azimuthal angle) around the center of the sample. In Appendix A 5, the scattering measurement is described in more detail.

\section{B. Artificial crossing of fiber bundles as a model system}

In real brain tissue, the underlying substructure and crossing angles of the nerve fibers are usually unknown. Therefore, we first test our measurement on a sample with a well-defined crossing angle. For this purpose, we extract two optic tracts from a $30 \mu \mathrm{m}$ section of a human optic chiasm [cf. Fig. 4(c)] and place them manually on top of each other with a crossing angle of about $80^{\circ}$ (see Fig. 8, top). The optic tracts contain mostly in-plane and parallel nerve fibers and are well suited as a model system for separate crossing fiber bundles.

The graphs in Fig. 8 show the average measured transmitted light intensity plotted against the angle of illumination for three selected regions: two regions with parallel
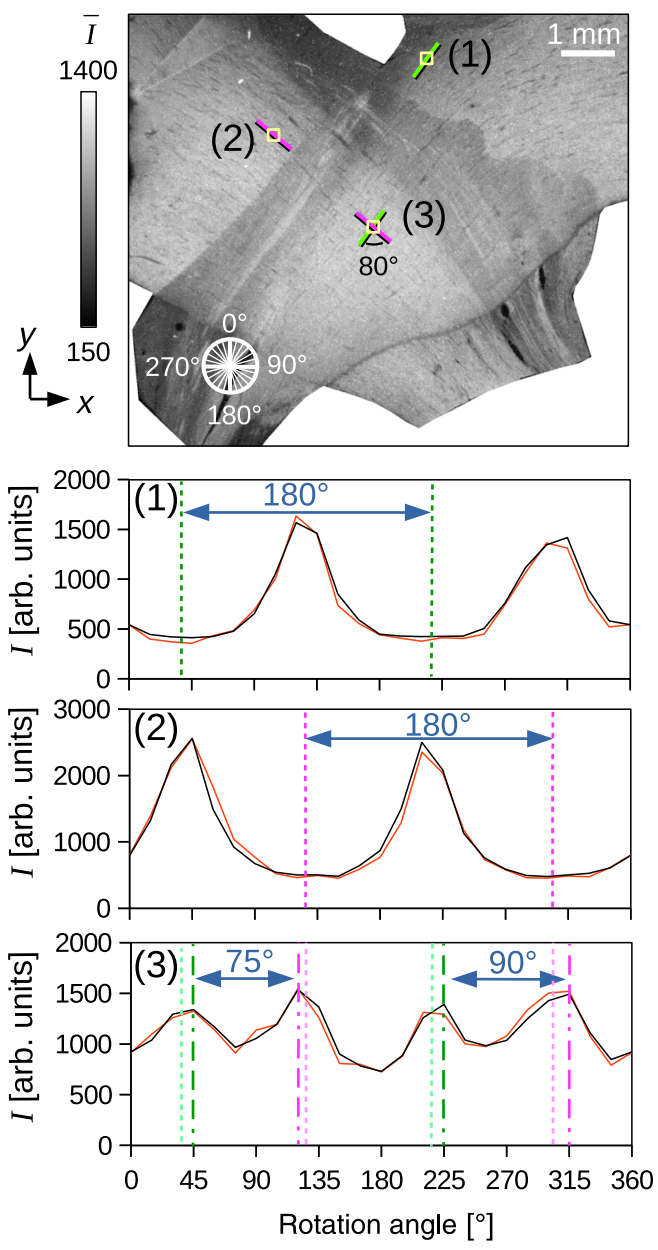

FIG. 8. Scattering measurement of two optic tracts, extracted from a $30-\mu \mathrm{m}$-thin section of a human optic chiasm, placed on top of each other with a crossing angle of approximately $80^{\circ}$. The measurement is performed as described in Appendix A 5 with a pixel size in object space of about $6.5 \mu \mathrm{m}$ and a mask with a rectangular hole which illuminates the sample under a fixed polar angle of $49.1^{\circ}$. During the measurement, the mask is rotated by angles of $\left\{0^{\circ}, 15^{\circ}, \ldots, 345^{\circ}\right\}$ around the center of the sample (starting on top and rotating clockwise; see the compass rose on top). The upper image shows the transmitted light intensity recorded by the camera averaged over all rotation angles of the mask. For evaluation, a region of $10 \times 10$ pixels is selected in each of the two fiber bundles [(1) and (2)] and in the crossing region (3). The selected regions are indicated by a yellow square in the upper image, and the in-plane orientations of the nerve fibers (derived from visible tissue structures) are indicated by green and purple lines. The graphs show the average transmitted light intensity $I$ in the three evaluated regions plotted against the rotation angle of the mask (black curves). The red curves show the transmitted light intensity of the middle pixel $(1 \times 1$ pixels $)$ in the selected regions. The dashed vertical lines indicate the orientations of the fibers as marked in the upper image. The dash-dotted lines in (3) indicate the position of the measured peaks.

fibers [(1) and (2)] and one region with crossing fibers (3). The yellow squares in the upper image mark the selected regions; the green and purple lines indicate the in-plane 
orientation of the nerve fibers (derived from visible structures). The black curves show the average transmitted light intensity in the selected regions $(10 \times 10$ pixels $)$, and the red curves the transmitted light intensity in a corresponding central pixel. The fact that the black and red curves are almost identical demonstrates the stability of our results and that the curve of a single image pixel can be used to determine the substructure of the corresponding tissue voxel (here, with an object-space resolution of $6.5 \mu \mathrm{m}$ per pixel).

During the scattering measurement, the nerve fibers light up when the direction of illumination is perpendicular to the fibers: For example, at a rotation angle of $0^{\circ}$ (illumination along the $y$ axis), fibers that are oriented perpendicular to the direction of illumination (along the $x$ axis) light up. Therefore, in regions with parallel in-plane fibers [(1) and (2)], the intensity curves show two distinct peaks that lie $180^{\circ}$ apart, and the position of the minima corresponds to the in-plane orientation of the optic tracts $\left(35^{\circ}\right.$ and $125^{\circ}$; see dashed vertical lines). In the crossing region (3), the intensity curve shows four peaks (peaks lying $180^{\circ}$ apart belong to one optic tract), and the distance between two neighboring peaks $\left(75^{\circ}-90^{\circ}\right)$ indicates the crossing angle between the two optic tracts

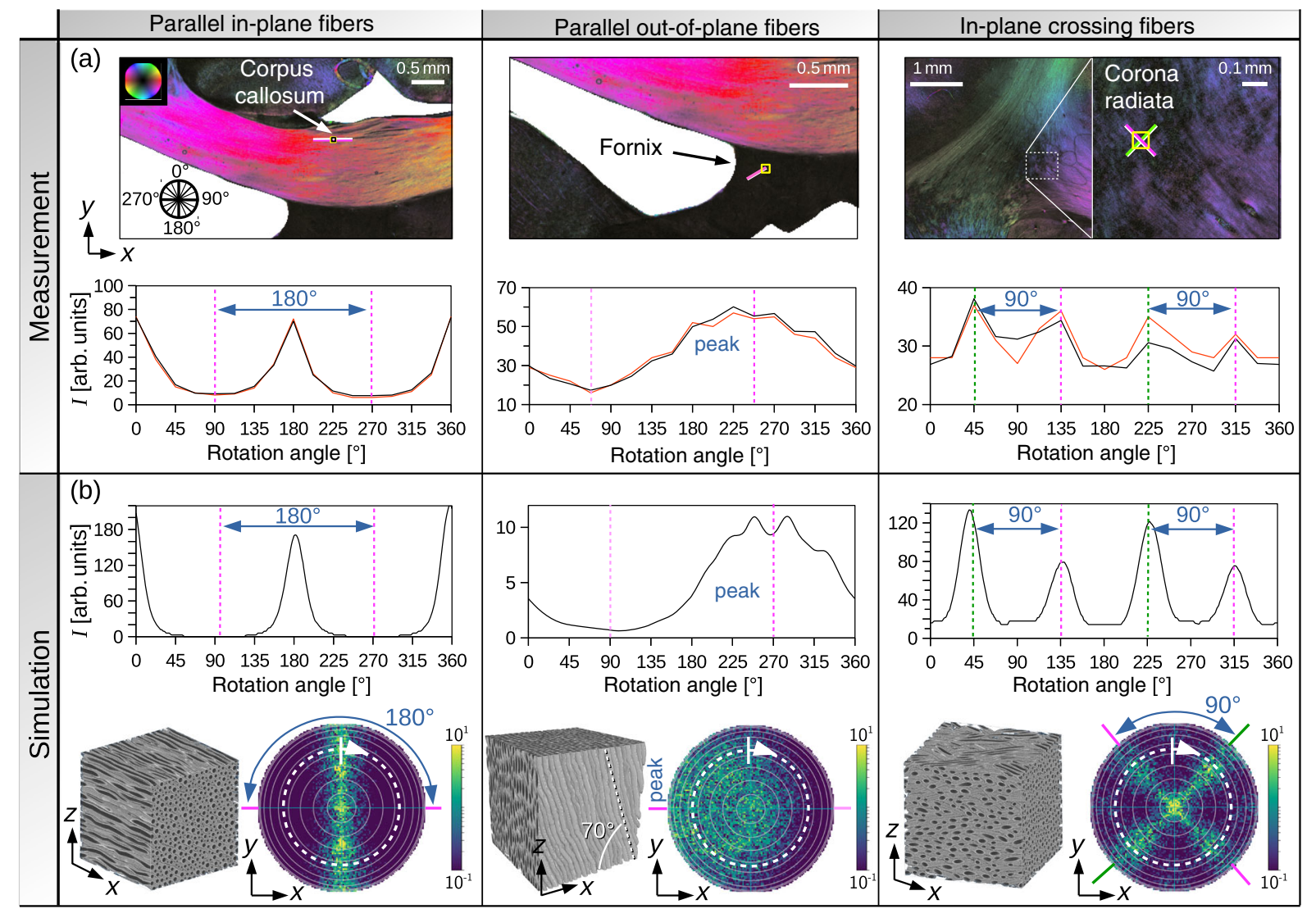

FIG. 9. Scattering measurement of a coronal vervet brain section in comparison to simulated scattering patterns. (a) The scattering measurement is performed as described in Appendix A 5 with a pixel size in object space of $6.3 \mu \mathrm{m}$ and a mask with a circular hole with a fixed polar angle of illumination of $47.6^{\circ}$ and rotation angles of $\left\{0^{\circ}, 22.5^{\circ}, \ldots, 337.5^{\circ}\right\}$. Three regions with parallel in-plane fibers (corpus callosum), parallel out-of-plane fibers (fornix), and in-plane crossing fibers (corona radiata) are selected for evaluation. The images in the top row show the fiber orientation maps obtained from a 3D-PLI measurement with $1.33 \mu \mathrm{m}$ pixel size (the fiber orientation map of the whole brain section is shown in Fig. 1). The selected regions $(10 \times 10$ pixels $)$ are marked by a small square, and the in-plane orientations of the nerve fibers (derived from visible structures and/or anatomical knowledge) are marked by purple and green lines. The second row shows the average transmitted light intensity $I$ in the evaluated regions plotted against the rotation angle of the mask (black curves). The red curves show the transmitted light intensity of the middle pixel $(1 \times 1$ pixels $)$ in the selected regions. The dashed vertical lines indicate the orientations of the fibers as marked in the fiber orientation maps. (b) The bottom row shows artificial fiber bundles and the simulated scattering patterns (cf. Fig. 7): parallel in-plane fibers, parallel out-of-plane fibers with $70^{\circ}$ inclination, and interwoven crossing fibers with a $90^{\circ}$ crossing angle. The green and purple lines around the scattering patterns indicate the main orientations of the artificial fibers. The white dashed circle indicates the angle under which the sample is illuminated in the measurement $\left(47.6^{\circ}\right)$. The graphs above show the intensity of the corresponding scattering pattern, evaluated along the dashed circle in the clockwise direction starting at the top (see the white arrow in the scattering pattern). To account for the finite size of the hole of illumination, a Gaussian blur with a diameter of $8^{\circ}$ in angular space is applied to the scattering patterns before evaluation. 
(approximately $80^{\circ}$ ). Taking into account that the scattering measurement is performed in steps of $15^{\circ}$, the measured crossing angle corresponds very well to the actual crossing angle of the specimen.

\section{Validation on known brain regions}

To demonstrate that the scattering measurement can be used to reveal the substructure of whole brain tissue samples, we measure a brain section with known anatomical structures [coronal vervet monkey brain section, shown in Figs. 1 and 2(a)] and compare the results to the simulated scattering patterns in Sec. IVA.

Three different anatomical structures are selected for evaluation: parallel in-plane fibers (corpus callosum), parallel out-of-plane fibers (fornix), and in-plane crossing fibers (corona radiata). Figure 9(a) shows the fiber orientation maps of the corresponding structures (upper images). The little yellow squares mark the evaluated regions $(10 \times 10$ pixels $)$; the purple and green lines indicate the in-plane orientation of the nerve fibers (derived from visible structures and/or anatomical knowledge). The graphs below show the average transmitted light intensity for each evaluated region plotted against the azimuthal angle of illumination (black curves, whole region; red curves, middle pixel). Again, the black and red curves are very similar to each other, demonstrating the stability of our results.

Just as observed for the two optic tracts (Sec. IV B), regions with in-plane nerve fibers light up when the direction of illumination is perpendicular to the fibers; i.e., the minimum of the intensity curves indicates the in-plane orientation of the fibers (see dashed vertical lines): In regions with parallel fibers (e.g., corpus callosum), the intensity curve shows two distinct peaks that lie $180^{\circ}$ apart [Fig. 9(a), left]; in regions with crossing fibers (e.g., corona radiata), the curve shows four peaks [peaks lying $180^{\circ}$ apart belong to one fiber bundle; see Fig. 9(a), right]. Regions with steep fibers show a different behavior: The intensity curve has a single broad peak, and the position of the peak coincides with the orientation of the fibers [see Fig. 9(a), middle].

Figure 9(b) shows the simulated scattering patterns for parallel in-plane fibers, parallel out-of-plane fibers with $70^{\circ}$ inclination, and interwoven crossing fibers with a $90^{\circ}$ crossing angle (cf. Fig. 7). To enable a comparison with the measured data, the scattering patterns are evaluated along the circle of illumination $\left(47.6^{\circ}\right.$ from the center; see the white dashed circle in the scattering pattern).

The resulting graphs in Fig. 9(b) correspond very well to the graphs obtained from the scattering measurements in Fig. 9(a), which demonstrates that our simulations make accurate predictions and that scattering measurements can indeed be used to extract valuable information about the substructure of brain tissue like the crossing angle of the nerve fibers.

\section{DISCUSSION}

\section{A. Transmittance measurements of brain sections}

When studying brain tissue properties, it should be noted that there exists a large variability between brains from different species, but also between brains from different subjects $[57,58]$. In addition, every brain section is unique in terms of its exact tissue composition and preparation. In contrast to the birefringence signal, the transmittance of brain tissue depends very much on these factors, causing differences in consecutive brain sections [cf. 3D-reconstructed volume of transmittance images in Figs. 13(b) and 13(c)]. For this reason, we combine various experimental studies on different species, subjects, and brain sections (Sec. III A) to demonstrate that the transmittance decreases steadily with an increasing inclination angle of the nerve fibers (by more than $50 \%$ for all investigated tissues) - independently from these factors. This finding suffices to perform the classification procedure of brain tissue presented in Sec. III C and to distinguish in-plane crossing from out-of-plane nerve fibers.

\section{B. Simulating light scattering in brain tissue}

To model and better understand the observed transmittance effects, we perform FDTD simulations using artificial nerve fiber models. In previous top-down simulation approaches of 3D-PLI, the birefringence of the nerve fibers has been modeled by series of Jones matrices $[36,56]$. In contrast to the birefringence, the transmittance of brain tissue is less determined by absorption (absorption coefficient is small [50,51]) and mostly by scattering of light, for which there exists to date no simple model. The FDTD simulations solve Maxwell's equations in a bottomup approach and require much more computing time, but they enable studies of complex light-matter interactions like scattering at micrometer resolution, without detailed knowledge of the scattering behavior of brain tissue. Therefore, we use FDTD simulations to develop a model for the transmittance of brain tissue and to improve the interpretation of measured data.

FDTD simulations are a proven tool for studying light scattering in lithography applications [59-61] or nanostructures [62-64]. They have also been applied to investigate microscopy measurements of nonbiological and biological tissue samples $[59,65,66]$ but not yet to brain tissue. One reason is that simulations of tissue samples that have dimensions of several micrometers and include all structural details are computationally too intense, because the mesh size in the simulation needs to be much smaller than the wavelength and should be small enough to resolve all geometrical features. To still enable the investigation of larger samples like brain tissue, we use high-performance computing and a simplified simulation model for the optics of the imaging system and the inner structure of the nerve fibers. 
Most nerve fibers in the brain are surrounded by a so-called myelin sheath, which consists of multiple layers with 3-5 $\mathrm{nm}$ thickness (see Appendix D). If the exact layered structure of the myelin sheath is modeled, the mesh size in the simulations can be at most $3 \mathrm{~nm}$. In this case, the simulation of a single nerve fiber with $1 \mu \mathrm{m}$ diameter consumes almost 290000 core hours (see Appendix H 1). To enable the simulation of larger tissue samples with various simulation parameters, we develop a simplified nerve fiber model with double myelin layers and a mesh size of $25 \mathrm{~nm}$.

Despite these simplifications, FDTD simulations are feasible to model sample sizes only up to the order of $100 \times 100 \times 100 \mu \mathrm{m}^{3}$, which is much smaller than the investigated brain sections (with diameters of several centimeters). However, as the in-plane resolution of the employed imaging systems and the nerve fiber diameters are on the order of $1 \mu \mathrm{m}$, the sample size used for the simulations $\left(30 \times 30 \times 30 \mu \mathrm{m}^{3}\right)$ is still sufficient to make predictions for different fiber configurations within a measured tissue voxel.

The FDTD simulations are computationally too expensive to simulate all different possible substructures. In order to develop general models that can be applied to the interpretation of brain tissue, the predictions from the simulations should not depend on the very details of the simulated substructure. Although the scattering of light depends on details of the simulated nerve fiber configurations like the axon diameter, myelin sheath thickness, or fiber orientation distribution [67], these dependencies are negligible compared to the influence of the fiber inclination (see Appendix H and Sec. III A 2).

\section{Validation of the simulation approach}

While the FDTD algorithm itself is an established method and has been proven to yield reliable results $[47,68]$, the validation of the simulation approach to correctly model brain tissue properties is not so straightforward. Artificial phantoms that provide similar dimensions and properties as nerve fibers are not available and cannot be used for validation. Therefore, we validate our simulation approach and the employed models in different ways.

To validate the model of the imaging system, the simulation algorithm was tested on a well-defined sample [U.S. Air Force (USAF) resolution target; see Appendix H 2 and Ref. [67] ]. The robustness of the simulation model with respect to changes in the simulation parameters (numbers of myelin layers, wavelengths, and mesh sizes) is validated in a rigorous study; see Appendix H. We show that the simplified models still reproduce the transmittance effects observed in the measurements (Sec. III A 2) and that our results are not sensitive to small changes in the simulation parameters, so our model is a good compromise between accuracy and computing time.
Finally, the predictions of our simulation algorithm are validated both experimentally and by using anatomical knowledge.

The experimental and simulation studies in Sec. III B are performed independently from each other and still yield the same result that the transmittance is mostly independent of the crossing angle. The validation of the classification procedure in Sec. III C is partially based on the predictions from the simulation studies and, therefore, also serves as an indirect validation of the simulation approach.

Most importantly, the scattering measurement is designed after analyzing the simulated scattering patterns. The measurement results correspond well to the simulated prediction, both in a well-defined model system (two crossing optic tracts, Sec. IV B) and in whole brain tissue samples (Sec. IV C). In particular, the model system has a very similar structure as the simulation model of separate crossing fiber bundles and can, therefore, serve as a tissue "phantom." The correspondence between experimental and simulation results provides compelling evidence that both our simulation algorithm and our experimental procedures work as intended.

\section{Generalization of the developed simulation framework}

The developed simulation framework can easily be adapted to microscopy techniques with different optics (numerical aperture, wavelength, polarization, etc.). Our framework is optimized for large-scale, complex fiber structures with dimensions in the micrometer scale, but our findings are not restricted to brain tissue. As the simulated samples are characterized only by their geometry and refractive indices, biological and nonbiological samples with comparable fibrous structures and refractive index differences (e.g., muscle fibers, collagen, and artificial fibers) are expected to show similar transmittance effects. (To increase the transmittance contrast between flat and steep fiber structures, the embedding solution should have a noticeably different refractive index than the fibers.) Scattering measurements revealing the fiber crossing angle could, for example, enhance the interpretation of collagen structures in the sclera or the lamina cribrosa of the eye $[69,70]$.

\section{E. Correction of misinterpreted 3D-PLI signals}

In Sec. III C, we present a classification procedure to correct for misinterpreted 3D-PLI signals. The classification can be applied to already existing 3D-PLI data, without a need for an advanced setup with a tiltable specimen stage. This feature allows an automated postprocessing of large image datasets, improving the reliability of 3D-PLI nerve fiber orientations in already existing brain atlases. By identifying misinterpreted regions with low fiber density or in-plane crossing fibers, the computed 3D fiber 
orientations in all other regions can be considered as reliable.

While tilting allows an improved interpretation of out-ofplane nerve fibers in regions with low fiber density (e.g., in the cortex), the employed model assumes parallel fibers and does not take fiber crossings into account $[42,43]$. With the developed classification procedure, we are now able to reliably identify regions with crossing nerve fibers, which can serve as a priori information for anatomical studies and tractography algorithms. From simulation studies, we know that the strength of the birefringence signal (retardation) decreases with an increasing crossing angle of the nerve fibers [56]. Future studies should investigate how the strength of the birefringence signal can be used to estimate the fiber crossing angle in regions with identified fiber crossings.

\section{F. Applications in conventional transmission microscopy}

Polarization-dependent light scattering which leads to diattenuation (polarization-dependent attenuation of light) cannot explain the observed inclination dependence of the transmittance, because the diattenuation of brain tissue is small $[71,72]$. In the present study, we show that the observed transmittance effect is mostly independent of the polarization [see Fig. 18(a)]. Therefore, simple transmission microscopy images, which usually provide only $2 \mathrm{D}$ information, can be used to distinguish out-of-plane from in-plane fiber structures-provided that the reference (region with in-plane fibers and maximum density, e.g., corpus callosum) is known. The analysis is possible without a need to change the experimental setup or to repeat measurements and adds important 3D information to the analysis of measured data. Moreover, our findings enable an automated segmentation of brain tissue into regions with white and gray matter, which is traditionally done by evaluating cell density distributions.

\section{G. Scattering measurements revealing tissue substructures}

In Sec. IVA, we show the great potential of scattering measurements to reveal the underlying substructure of a measured tissue voxel, e.g., the crossing angle of the nerve fibers. Similar to how x-ray crystallography with a wavelength of about one angstrom can be used to reconstruct the structure of a crystal at molecular resolution (in the order of nanometers) [73], light in the visible spectrum $(\lambda \sim 0.5 \mu \mathrm{m})$ that is scattered when passing through a brain section contains information about the substructure of the tissue at the resolution of single fibers (in the order of micrometers).

The present study is a proof of concept, intended to validate the simulated scattering patterns and to show that it is indeed possible to obtain valuable additional information by measuring the scattering patterns of brain tissue. We use a prototype setup with limited spatial resolution $(\geq 6.3 \mu \mathrm{m}$ per pixel) and oblique illumination with a limited number of angles $\left(\geq 15^{\circ}\right.$ steps). Despite its simplicity, we are able to use the employed setup to reveal highly complex nerve fiber structures like crossing fibers in the corona radiata (see Sec. IV C). To resolve more details in the scattering pattern, e.g., in the case of fibers with small crossing angles or multiple crossings, the spatial and angular resolutions can easily be improved. By using more advanced techniques, e.g., by rotating the sample and light source or moving the detector behind the sample, it would be possible to measure the complete scattering pattern, gaining even more information about the underlying substructure, for example, the tissue homogeneity.

The FDTD simulations are essential to develop a model for the correct analysis of the scattering measurements. Once the model has been developed, it can be used for the analysis (e.g., in-plane fibers with different crossing angles) without a need for further simulations or computing time. When extracting different information from the scattering patterns (e.g., information about tissue homogeneity), further improvements of the model are needed. Future studies should address an automated evaluation of the scattering measurements.

The setup used for the scattering measurements can easily be integrated in the 3D-PLI setup, enabling studies of both scattering and birefringence on the same brain section without moving the sample, and the development of a more realistic model of the $3 \mathrm{D}$ nerve fiber architecture in the brain-also in regions with crossing fibers.

\section{CONCLUSION}

In this paper, we perform comprehensive experimental and simulation studies to investigate how light scattering in microscopy measurements can be leveraged to obtain additional information about the 3D structure of fibrous tissue samples like brain tissue. We show how misinterpreted signals in 3D-PLI can be corrected and present a new measurement technique that reveals nerve fibers crossings at the micrometer scale, allowing a reliable, high-resolution reconstruction of the brain's nerve fiber architecture.

First of all, we developed and successfully applied a versatile simulation framework based on the FDTD method. Using high-performance computing and a sophisticated simulation model, our tool enables one for the first time to use FDTD simulations to study transmission microscopy measurements, in particular, light scattering, on large-scale complex structures like brain tissue. We demonstrated that our simulations make valid predictions, provide explanations for effects observed in the measurement, and enable the development of new techniques that enhance the interpretation of the measured data and extract new information. The simulation framework can easily be generalized to other microscopy techniques with different optics and to tissue samples with comparable fibrous structures and refractive indices (e.g., muscle fibers, 
collagen, and artificial fibers), allowing applications beyond neuroscience.

Using extensive experimental studies on brain tissue (ranging from rodents and monkeys to humans) and FDTD simulations, we developed two major advances how the high-resolution reconstruction of nerve fibers, in particular, nerve fiber crossings, can be improved.

(i) 3D-PLI provides information about the $3 \mathrm{D}$ nerve fiber orientations at micrometer resolution. However, regions with in-plane crossing fibers were so far misinterpreted as out-of-plane fibers. We could demonstrate that with a combined analysis of transmittance and retardation (strength of the birefringence signal), it is possible to distinguish between these regions, allowing a more reliable reconstruction of nerve fiber orientations in the brain. A major advantage of this method is that it can be applied to past 3D-PLI measurements and correct misinterpreted nerve fiber orientations in large datasets without the need to repeat any measurements. Our findings can also be used to distinguish between in-plane and out-of-plane fibers in simple transmission microscopy images, adding important 3D information to the analysis of measured data, and to perform an automated segmentation of brain tissue into white and gray matter regions. As 3D-PLI reconstructs the 3D nerve fiber architecture with microscopic resolution, the knowledge about the existence of fiber crossings already significantly improves the reconstruction of the highly complex nerve fiber architecture in the brain.

(ii) We show that light scattering in the visible spectrum reveals the substructure of an image voxel, i.e., the crossing angle of the nerve fibers, with micrometer resolution. Our findings open up a new field of research: Scattering measurements can be used to obtain an even more detailed reconstruction of nerve fiber crossings in the brain and to solve the ongoing debate about the organization principles of crossing fibers. The improved reconstruction of nerve fiber crossings leads to an improved nerve fiber tractography, and thus not only to a better understanding of the structural organization principles in the brain, but also to a better interpretation of clinical data obtained from diffusion magnetic resonance imaging.

\section{ACKNOWLEDGMENTS}

We thank Markus Cremer, Christian Rademacher, and Patrick Nysten for the preparation of the histological brain sections, David Gräßel and Isabelle Mafoppa Fomat for the 3D-PLI measurements, Mike Huwer and David Gräßel for the scattering measurements, Philipp Schlömer for generating the transmittance and retardation images, David Gräßel for generating the fiber orientation maps, Martin Schober, Marcel Huysegoms, and Sascha Münzing for image registration, Felix Matuschke for developing the algorithms to generate the fiber configurations, Sebastian Bludau for the bright-field transmission microscopy measurements, Andreas Wree for providing the human occipital lobe, Roxana Kooijmans and the Netherlands Brain Bank for providing the human optic chiasm, and Karl Zilles and Roger Woods for collaboration in the vervet brain project. This work has received funding from the Helmholtz Association portfolio theme Supercomputing and Modelling for the Human Brain, from the European Union's Horizon 2020 Research and Innovation Program under Grant Agreements No. 7202070 and No. 785907 (Human Brain Project SGA1 and SGA2), and from the National Institutes of Health under Grant Agreements No. R01MH092311 and No. 5P40OD010965. We gratefully acknowledge the computing time granted through JARA-HPC on the supercomputer JURECA [74] and JUQUEEN [75] at Forschungszentrum Jülich. M. M. designed the study with help from M. A. and K. M., analyzed the measurements, and carried out the simulations. M. M., M. A., H. D. R., and K. M. contributed to the interpretation of the data and provided theoretical considerations. H. D. R. and K. M. provided the FDTD software. I. C., L. S., and F. S. P. produced the TPFM measurements. K. A. contributed to the anatomical content of the study. M. M. wrote the paper with revisions from M. A., H. D. R., K. A., and K. M.

\section{APPENDIX A: MEASUREMENT METHODS}

\section{Preparation of brain sections}

The measurements are performed on sections from human brains (Fig. 8: female, 74 yr old; Fig. 12: male, $87 \mathrm{yr}$ old), as well as on brain sections from vervet monkeys (African green monkey: Chlorocebus aethiops sabaeus, male, between 1 and 2 yr old), rats (Wistar, male, 3 months old), mice (C57BL/6, male, 6 months old), and a hooded seal [56]. All animal procedures have been approved by the institutional animal welfare committee at Forschungszentrum Jülich $\mathrm{GmbH}$, Germany, and are in accordance with European Union (National Institutes of Health) guidelines for the use and care of laboratory animals. The human brains are acquired in accordance with the local ethic committee of the University of Rostock, Germany, and the Netherlands Brain Bank, in the Netherlands Institute for Neuroscience, Amsterdam. A written informed consent of the subjects is available. The brains are removed from the skull within $24 \mathrm{hr}$ after death, immersed in a buffered solution of $4 \%$ formaldehyde for several weeks, immersed for several days in solutions of $10 \%$ and $20 \%$ glycerin combined with 2\% Dimethyl sulfoxide for cryoprotection, dipped in cooled isopentane for several minutes, and deeply frozen. The frozen brains are cut with a cryostat microtome (Leica Microsystems, Germany) at a temperature of $-30{ }^{\circ} \mathrm{C}$ into sections of $60 \mu \mathrm{m}$. (The human optic chiasm shown in Fig. 8 is cut into sections of $30 \mu \mathrm{m}$.) The brain sections are mounted on cooled glass slides, embedded in $20 \%$ glycerin solution, covered by a cover glass, sealed with lacquer, and weighted 
for several hours to prevent the development of air bubbles. The sections are measured 1-2 days after embedding.

\section{3D-PLI measurement}

The 3D-PLI measurements are performed with a highresolution polarizing microscope $(P M)$ manufactured by Taorad GmbH, Germany. The microscope has been used in previous 3D-PLI studies to measure the three-dimensional nerve fiber orientations at a high resolution $[34,35,41,76]$. The light source consists of a single white LED (IntraLED $2020+$ operated at $24 \mathrm{~W}$ ) with integrated Köhler illumination and a bandpass filter, generating a wavelength spectrum $\lambda=(550 \pm 5) \mathrm{nm}$. Further components are a rotatable linear polarizer, a specimen stage, a circular analyzer (quarter-wave retarder combined with linear polarizer), and a CCD camera (monochrome RETIGA4000R camera by QImaging with a Kodak KAI-04022ABA image sensor) which records an image for each rotation angle $\rho=\left\{0^{\circ}, 10^{\circ}, \ldots, 170^{\circ}\right\}$ of the polarizer, yielding a series of 18 images. The microscope is equipped with a motorized specimen stage (Märzhäuser, Germany) which performs a translational scan of the brain section in tiles of $2.7 \times 2.7 \mathrm{~mm}^{2}$. To allow for stitching, the tiles are measured with an overlap of $30 \%$ on all sides. The objective lens (Nikon TL Plan Fluor EPI P $5 \times$ ) has a $5 \times$ magnification and a numerical aperture of 0.15 . The resolution in object space is about $1.33 \mu \mathrm{m}$ per pixel.

The transmittance and retardation images are computed as described by Axer et al. [34,35] by performing a discrete harmonic Fourier analysis on the measured light intensities $I(\rho)$ per image pixel: $I(\rho)=a_{0}+a_{2} \cos (2 \rho)+b_{2} \sin (2 \rho)$. The transmittance $I_{T}$ corresponds to the average over all 18 images and is computed from the Fourier coefficient of order zero $\left(I_{T}=2 a_{0}\right)$; the retardation $|\sin \delta|$ corresponds to the amplitude of the intensity signal and is computed from the Fourier coefficients of order zero and two [ $|\sin \delta|=$ $\left.\left(a_{2}^{2}+b_{2}^{2}\right)^{1 / 2} / a_{0}\right]$, where $\delta$ is the phase shift induced by the birefringent brain tissue. The transmittance images are normalized by the transmittance image measured without a sample, yielding normalized transmittance images $\left(I_{T, N}\right)$.

Images of several consecutive brain sections (Fig. 13 in Appendix B 2) are registered onto each other using inhouse-developed software tools based on the software packages ITK, ELASTIX, and ANTs [77-81], which perform linear and nonlinear transformations. As an undistorted reference volume, aligned blockface images are used: A picture of the brain block surface (blockface image) is taken every time before sectioning, and a pattern of ARTag markers [82] is used to determine the position of the brain block in two-dimensional space [83].

\section{TPFM measurement}

The TPFM measurements are performed with a custommade two-photon fluorescence microscope $[29,44]$ at the
European Laboratory for Non-Linear Spectroscopy (LENS), University of Florence, Italy. The microscope is equipped with a mode-locked titanium-sapphire laser with a wavelength of $800 \mathrm{~nm}$ which is coupled into a scanning system based on a pair of galvanometric mirrors. The laser is focused onto the sample by a water-immersion $25 \times$ objective lens (LD LCI Plan-Apochromat $25 \times / 0.8 \mathrm{Imm}$ Corr DIC M27). The lateral displacement of the sample is realized by a motorized $x y$ stage (enabling tilewise scanning of the sample). The axial displacement (along the $z$ axis) is realized by a closed-loop piezoelectric stage. The fluorescence signals are collected by two photomultiplier tubes, which detect red and green fluorescence. The setup achieves a resolution of $0.244 \times 0.244 \times 1 \mu \mathrm{m}^{3}$. The sample is measured in tiles of $250 \times 250 \mu \mathrm{m}^{2}$, with an overlap of $10 \%$ to allow for stitching.

\section{Bright-field transmission microscopy}

The bright-field transmission microscopy images (Fig. 12 in Appendix B 1 and Fig. S1 in Supplemental Material [55]) are obtained from ZEISS Axio Imager Vario. The microscope is equipped with a white microLED, which emits unpolarized light with wavelengths between 400 and $750 \mathrm{~nm}$. The objective lens (Plan Apochromat 5x) has a $5 \times$ magnification and a numerical aperture of 0.16 . The resolution in object space is about $0.91 \mu \mathrm{m}$ per pixel.

\section{Light scattering measurement}

The scattering measurements are performed with the setup shown in Fig. 10. The customized light source (FZJSQ300-DL-G-WCO provided by LUMIMAX ${ }^{\circledR}$, iiM AG, Germany) consists of a matrix of $36 \times 36$ LED diodes (NSPG 510S, Nichia Corporation) and a diffuser plate (acrylic glass), which illuminate an area of approximately $30 \times 30 \mathrm{~cm}^{2}$ and generate mostly incoherent and unpolarized light with a wavelength of $(525 \pm 25) \mathrm{nm}$.

A mask with a hole is placed on top of the light source so that the sample is illuminated under an angle around $47^{\circ}-49^{\circ}$ [see Fig. 10(a)]. The transmitted light intensity behind the sample is recorded by a CCD camera (AVT Oscar F-810C, sensor ICX-456AQ, objective lens ApoRodagon-N 4.0/90, focal length $90 \mathrm{~mm}$ ), which is placed above the sample at a sufficient distance to ensure that only light with nearly perpendicular incidence is collected. During the measurement, the mask is rotated around the center in equidistant steps in the clockwise direction, and an image is recorded for each rotation angle. To achieve a sufficient signal-to-noise ratio, a long exposure time $(0.5-2 \mathrm{sec})$ is chosen.

For the scattering measurement of the coronal vervet brain section in Sec. IV C, a mask with a circular hole [diameter $3.5 \mathrm{~cm}$, distance from center $11.5 \mathrm{~cm}$; see Fig. 10(b)] is used. The distance between the light source 
(a)

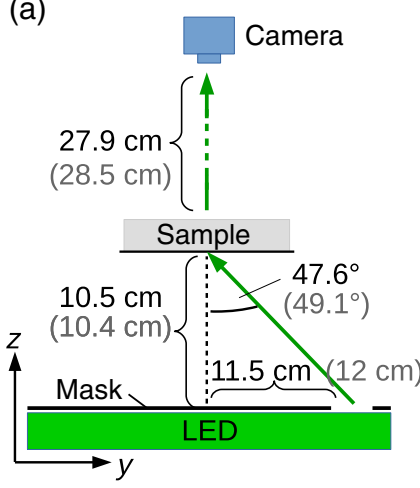

(b)

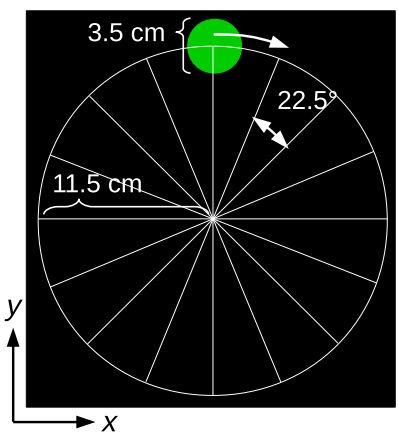

FIG. 10. Schematic setup of the scattering measurement. (a) Side view of the setup, consisting of an LED panel with a mask, a specimen stage with a sample, and a camera. The numbers without brackets belong to the scattering measurement of the vervet brain section (Sec. IV C), and the numbers in brackets to the measurement of the human optic chiasm (Sec. IV B). (b) Top view of the mask used for the scattering measurement in Sec. IV C (circular hole with $3.5 \mathrm{~cm}$ diameter and $11.5 \mathrm{~cm}$ distance from the center, yielding an illumination angle of $47.6^{\circ}$ with respect to the center of the sample). During the measurement, the mask is rotated in steps of $22.5^{\circ}$ around the center in the clockwise direction. [For the scattering measurement in Sec. IV B, a mask with a rectangular hole $\left(2.4 \times 4 \mathrm{~cm}^{2}\right)$ and steps of $15^{\circ}$ are used.].

and sample is set to $10.5 \mathrm{~cm}$ so that the center of the hole illuminates the center of the sample under an angle of $47.6^{\circ}$ [see Fig. 10(a)]. The distance between the camera objective and sample is set to $27.9 \mathrm{~cm}$, yielding a pixel size in object space of about $6.3 \mu \mathrm{m}$. During the measurement, the mask is rotated in steps of $22.5^{\circ}$ with an exposure time of $0.5 \mathrm{sec}$.

For the scattering measurement of the human optic chiasm in Sec. IV B, a mask with a rectangular hole $\left(2.4 \times 4 \mathrm{~cm}^{2}\right.$, distance from center $\left.12 \mathrm{~cm}\right)$ is used. The distance between the light source and sample is set to $10.4 \mathrm{~cm}$ so that the center of the hole illuminates the center of the sample under an angle of $49.1^{\circ}$ [see Fig. 10(a)]. The distance between the camera objective and sample is set to $28.5 \mathrm{~cm}$, yielding a pixel size in object space of about $6.5 \mu \mathrm{m}$. During the measurement, the mask is rotated in steps of $15^{\circ}$ with an exposure time of $2 \mathrm{sec}$.

\section{APPENDIX B: TRANSMITTANCE MEASUREMENTS OF INCLINED FIBERS}

\section{Transmittance of flat and steep nerve fibers}

In large anatomical structures with densely packed nerve fibers, the inclination angles of the nerve fibers cannot be exactly determined by 3D-PLI or TPFM measurements. By investigating sections from brains that are cut along mutually orthogonal anatomical planes, the transmittance of the same brain region can be evaluated for flat nerve fibers (with inclination angles $\alpha<45^{\circ}$ ) in one section plane and for steep nerve fibers $\left(\alpha>45^{\circ}\right)$ in the other section plane. Since different brain sections from different specimens might not be comparable due to differences in the tissue structure and preparation, the transmittance values are compared only within the same brain section.

Figure 11 shows the normalized transmittance images $I_{T, N}$ of coronal and sagittal sections from rat and vervet monkey brains. The coronal (sagittal) section planes are indicated by blue (red) lines in the respective other brain section for reference. Selected brain structures are identified according to rat $[14,84,85]$ and vervet [52-54] brain atlases. The transmittance values are evaluated in brain
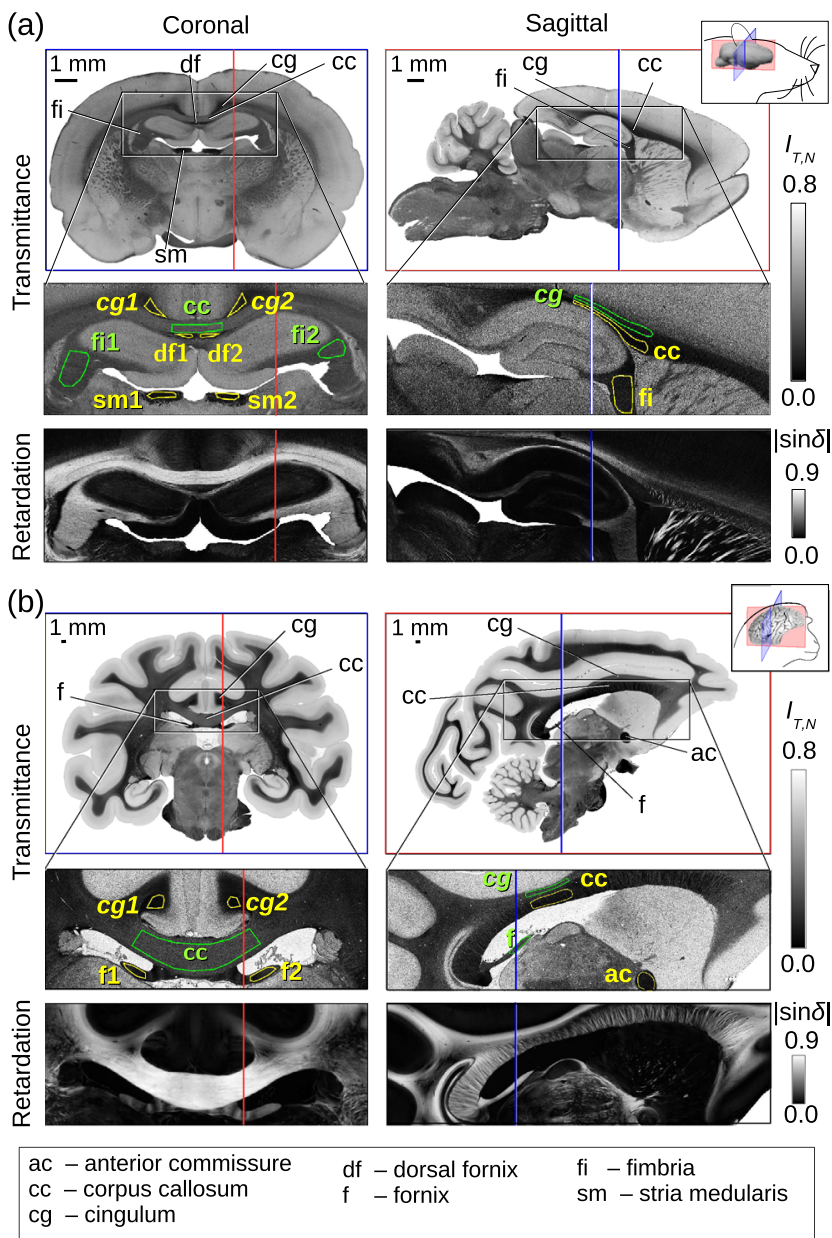

FIG. 11. Transmittance and retardation images of coronal and sagittal brain sections for a rat (a) and a vervet monkey (b). The coronal (sagittal) section planes are indicated by blue (red) lines in the respective other brain section for reference, and selected anatomical structures are labeled (see the legend). The upper two rows of each panel show the normalized transmittance images $I_{T, N}$, and the third row shows the retardation images $|\sin \delta|$, both obtained from 3D-PLI measurements with $1.33 \mu \mathrm{m}$ pixel size. The images in the first row show the whole brain section, and the images in the second and third rows show an enlarged view. The selected regions in yellow (green) belong to steep (flat) nerve fibers, which appear dark (bright) in the transmittance and retardation images. 
TABLE I. Mean transmittance values $\left(\overline{I_{T, N}}\right)$ and standard deviation for the selected green and yellow areas in Fig. 11. Areas belonging to the same structure are evaluated together $(\operatorname{cg} \equiv \operatorname{cg} 1 \cup \operatorname{cg} 2)$.

\begin{tabular}{lccccc}
\hline \hline & \multicolumn{2}{c}{ Rat } & & \multicolumn{2}{c}{ Vervet } \\
\cline { 2 - 3 } \cline { 5 - 6 } & Coronal & Sagittal & & Coronal & Sagittal \\
\hline ac & $\ldots$ & $\cdots$ & $\ldots$ & $0.11 \pm 0.04$ \\
cc & $0.33 \pm 0.16$ & $0.09 \pm 0.04$ & $0.24 \pm 0.09$ & $0.11 \pm 0.05$ \\
cg & $0.15 \pm 0.06$ & $0.18 \pm 0.09$ & $0.08 \pm 0.02$ & $0.24 \pm 0.09$ \\
df & $0.10 \pm 0.03$ & $\cdots$ & $\cdots$ & $\cdots$ \\
f & $\cdots$ & $\ldots$ & $0.13 \pm 0.06$ & $0.26 \pm 0.10$ \\
fi & $0.25 \pm 0.10$ & $0.15 \pm 0.07$ & $\cdots$ & $\cdots$ \\
sm & $0.08 \pm 0.03$ & $\cdots$ & $\cdots$ & $\cdots$ \\
\hline \hline
\end{tabular}

regions that have a relatively homogeneous tissue composition and that include predominantly flat nerve fibers (areas surrounded in green) or steep nerve fibers (areas surrounded in yellow).

The approximate orientation of the nerve fibers is known from the anatomy of the rat and the vervet brain as described in the atlases and is confirmed by the retardation images $|\sin \delta|$ shown below the transmittance images in Fig. 11: Regions with flat (noncrossing) nerve fibers are expected to show larger retardation values than regions with steep nerve fibers $\left(\delta \propto \cos ^{2} \alpha\right)$. The mean transmittance values and the standard deviation for the evaluated green and yellow areas can be found in Table I.

In regions with flat nerve fibers, the mean transmittance values are larger $\left(\overline{I_{T, N}} \in[0.18,0.33]\right)$ than in regions with steep nerve fibers $\left(\overline{I_{T, N}} \in[0.08,0.15]\right)$. A region with flat (steep) nerve fibers which shows large (small) transmittance values in one section plane (coronal or sagittal) shows the opposite behavior in the corresponding orthogonal section plane. The difference is especially large when comparing the transmittance values of the corpus callosum (a massive fiber tract connecting the two hemispheres of the brain) and the cingulum (a $\mathrm{C}$-shaped fiber structure running mostly perpendicular to the corpus callosum). In the coronal brain sections, the fibers of the cingulum $(\mathrm{cg})$ run mostly perpendicular to the section plane and have about 55\%-67\% lower transmittance values than the fibers of the corpus callosum (cc) which lie mostly within the section plane. In the sagittal brain sections, the situation is exactly the opposite: The transmittance values of the corpus callosum are about 50\%-54\% less than the transmittance values of the cingulum. Fibers in the rat and vervet monkey brains show a similar pattern.

Images obtained from conventional bright-field transmission microscopy with unpolarized light show similar effects, not only in vervet but also in human brain sections (see Fig. 12 and Fig. S1 in Supplemental Material [55]): Regions with steep (out-of-plane) nerve fibers appear darker than regions with flat (in-plane) nerve fibers.

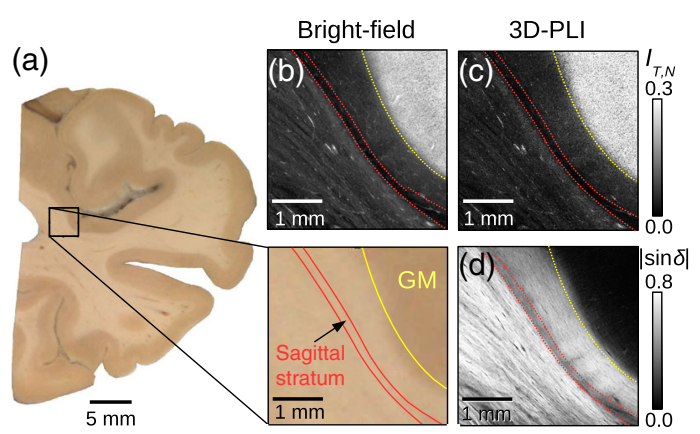

FIG. 12. Bright-field transmission microscopy vs 3D-PLI measurement of a human brain section (right occipital lobe). (a) Photograph of the brain block surface before sectioning (blockface image). The enlarged region shows the sagittal stratum, a white matter structure that runs mostly perpendicular to the section plane ( $\mathrm{GM}=$ gray matter). (b) Bright-field transmission microscopy image of the same region with $0.91 \mu$ m pixel size. (c) Normalized transmittance image of the same region obtained from a 3D-PLI measurement with $1.33 \mu \mathrm{m}$ pixel size. (d) Corresponding retardation image. Regions with steep out-of-plane fibers (sagittal stratum) show lower transmittance (and retardation) values than the neighboring regions with nonsteep fibers; the transmitted light intensity images obtained from bright-field transmission microscopy (b) and 3D-PLI (c) look similar.

\section{3D reconstruction of transmittance images}

So far, we have compared single brain sections from different species to each other. To study the transmittance across several consecutive brain sections, we register the transmittance images of 234 coronal sections from the right hemisphere of a vervet monkey brain onto each other using in-house-developed software tools (see Appendix A2). Figure 13 shows the 3D-reconstructed transmittance images along three orthogonal anatomical planes: coronal (a), sagittal (b), and horizontal (c), as well as a detail of the 3D volume (d). The white arrows point to the sagittal stratum-a white matter structure with nerve fibers that are oriented mostly perpendicular to the image plane (along the $z$ direction), as can be seen in the sagittal and horizontal planes. The structure appears much darker in the transmittance images than the surrounding regions. Thus, the observation that steep nerve fibers show lower transmittance values than flat nerve fibers is consistent across several consecutive brain sections.

\section{Transmittance contrast of nerve fiber bundles in mutually orthogonal planes}

In bulk tissue with densely packed nerve fibers, the fiber inclinations cannot be exactly determined. In regions with distinct fiber bundles, however, the inclination angles can be estimated by manually evaluating the course of the fiber bundles in different section planes. For this purpose, we select a structure in the rat brain that contains several distinct nerve fiber bundles with different, well-defined inclination angles - the so-called caudate putamen. To estimate the inclination angles of the nerve fibers, we evaluate 

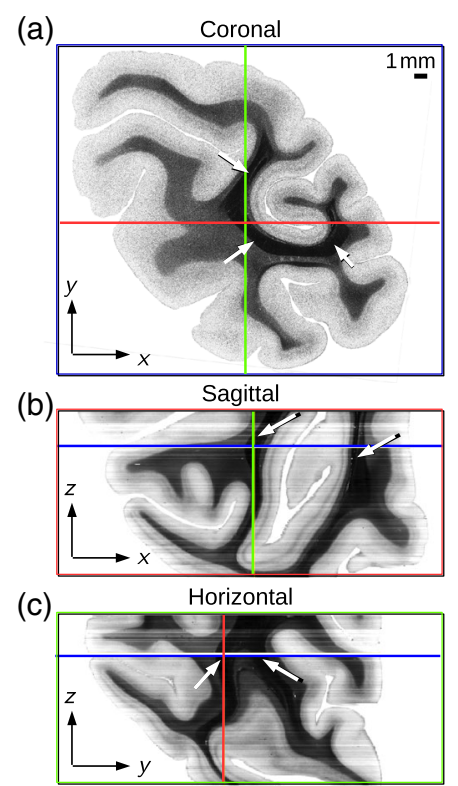

(d)

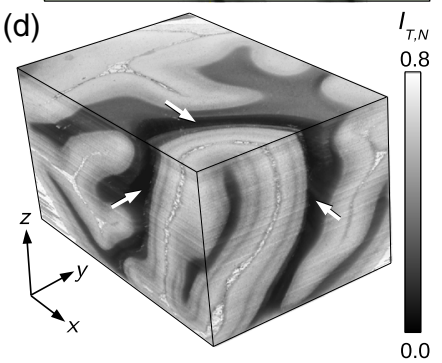

FIG. 13. 3D-reconstructed normalized transmittance images $\left(I_{T, N}\right)$ of the right hemisphere of a vervet monkey brain (234 consecutive sections from the occipital lobe) obtained from 3DPLI measurements with $1.33 \mu \mathrm{m}$ pixel size. The brain is cut along the coronal plane ( $x y$ plane), and the resulting brain sections are registered onto each other in the $z$ direction. (a)-(c) Cross sections of the $3 \mathrm{D}$ volume shown along the coronal ( $x y$ ), sagittal $(x z)$, and horizontal $(y z)$ planes. The colored lines indicate the position of the displayed $x y, x z$, and $y z$ planes. (d) Detail of the $3 \mathrm{D}$ volume. The white arrows point to the sagittal stratum-a white matter structure that runs mostly perpendicular to the image plane (along the $z$ direction) and which appears much darker in the transmittance images than the surrounding tissue.

the course of the bundles in mutually orthogonal section planes (coronal, sagittal, and horizontal); see Fig. 14(a). As the brain sections are obtained from different brains and might differ in tissue composition and preparation, the transmittance images cannot be directly compared to each other. To still enable a comparison between the transmittance of flat and steep nerve fibers, the transmittance values in regions with fibers are compared to the transmittance values in regions with surrounding tissue for each brain section separately, assuming that the transmittance of the surrounding tissue does not depend much on the choice of the section plane.

To separate the fiber bundles from the surrounding tissue, we use the image contrast of the retardation images [see the yellow lines in Fig. 14(b)]. Figure 14(c) shows the corresponding histograms of the transmittance evaluated in regions with nerve fibers (pink) and in regions with surrounding tissue (cyan). As the coronal brain section contains mostly steep nerve fibers which yield low retardation values, the image contrast in the retardation image is not large enough to separate the fibers from the surrounding tissue. Therefore, we compute the histogram for the whole caudate putamen (area surrounded by yellow line) and assume that the peak with lower (larger) transmittance belongs to nerve fibers (surrounding tissue).

From the minimum and maximum peak transmittance values of the histograms [pink and cyan numbers in Fig. 14(c)], we compute the transmittance contrast $\mathcal{C} \equiv$ $\left(I_{T, \text { max }}-I_{T, \text { min }}\right) /\left(I_{T, \text { max }}+I_{T, \text { min }}\right)$ between fiber bundles and surrounding tissue. For flat fiber bundles in the sagittal and horizontal brain sections, this contrast is much lower $\left(5^{\circ} \leq \alpha \leq 60^{\circ}: \mathcal{C} \approx 14 \%-20 \%\right)$ than for steep fiber bundles in the coronal brain section $\left(45^{\circ} \leq \alpha \leq 85^{\circ}: \mathcal{C} \approx 62 \%\right)$. Assuming that the transmittance of the surrounding tissue is mostly independent of the fiber orientation, this result demonstrates again that the transmittance values for steep nerve fibers are significantly lower than for flat nerve fibers.

\section{Transmittance vs inclination of nerve fiber bundles in TPFM images}

The studies described in Appendices B 1-B 3 have been performed without knowing the exact inclination angles of the nerve fibers. To determine the fiber inclination, we measure the caudate putamen of a coronal mouse brain section both with 3D-PLI and with TPFM [see the inset in Fig. 3(a) and Fig. S2 in Supplemental Material [55] ].

To obtain the inclination angles of the fiber bundles, the cross sections of the bundles are determined in the first and the last slice of the TPFM image stack [cf. Fig. S2(d) [55] ]. For each fiber bundle, the inclination angle is computed from the midpoints of the corresponding cross sections and from the thickness of the brain section [cf. Fig. S2(c) [55] ]. The fiber inclination and transmittance values are evaluated for 40 fiber bundles in the caudate putamen [see the colored shapes in Figs. S2(b) and S2(d) [55] ].

The scatter plot in Fig. 3(a) shows the averaged transmittance values plotted against the determined fiber inclination angles. Although the values are broadly distributed, the scatter plot shows a clear tendency toward a decrease in transmittance with an increasing fiber inclination angle. The values in orange belong to regions with lower fiber densities which might lead to overestimated transmittance values. However, the decrease in transmittance is also observed in regions with maximum fiber density (values in blue): While the mean transmittance values for flat nerve fibers $\left(\alpha<50^{\circ}\right)$ reach larger values $\left(0.1<\overline{I_{T, N}}<0.2\right)$, the mean transmittance values for steep nerve fibers $\left(\alpha>60^{\circ}\right)$ are small $\left(\overline{I_{T, N}}<0.05\right)$. 


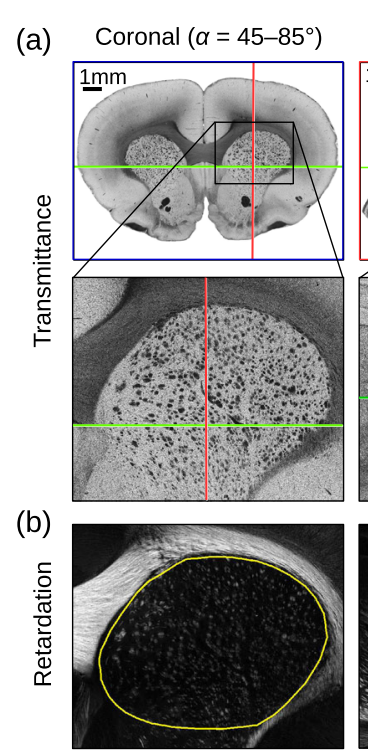

(c)

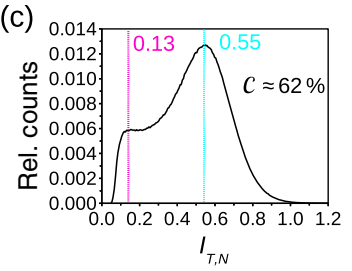

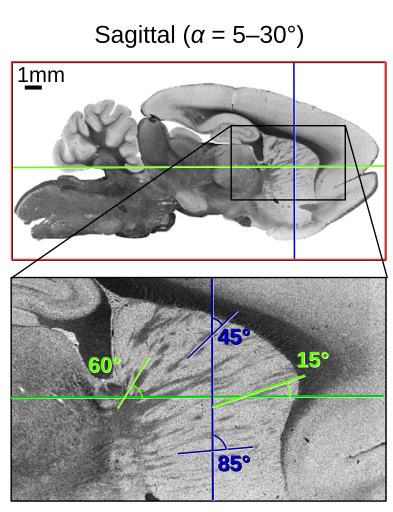
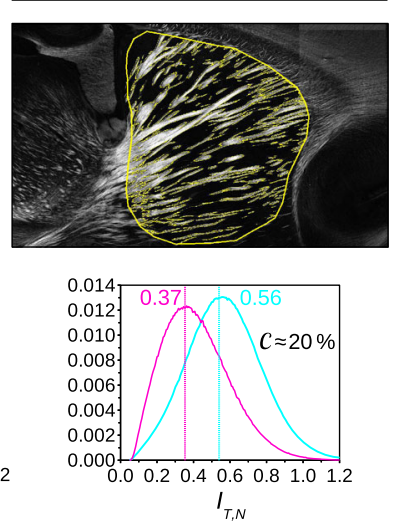
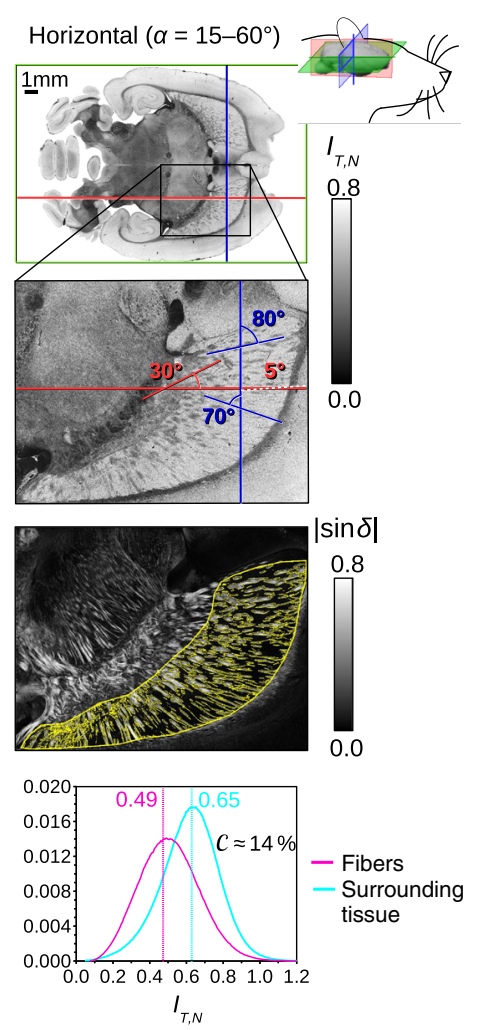

FIG. 14. Transmittance contrast of nerve fiber bundles in mutually orthogonal anatomical planes. (a) Normalized transmittance images $\left(I_{T, N}\right)$ of a coronal, sagittal, and horizontal rat brain section obtained from 3D-PLI measurements with $1.33 \mu \mathrm{m}$ pixel size. The colored lines indicate the approximate position of the section planes. The enlarged views show the region of the caudate putamen and the maximum and minimum angles under which the nerve fiber bundles are oriented with respect to the section planes. (b) Corresponding retardation images $(|\sin \delta|)$ of the enlarged views. The image contrast is used to select regions with fibers and with surrounding tissue in the caudate putamen (yellow lines). (c) Histograms of the transmittance values $\left(I_{T, N}\right)$ for the selected regions with nerve fibers (pink) and with surrounding tissue (cyan) in the caudate putamen. For the coronal brain section, the retardation image cannot be used to separate the fibers from the surrounding tissue, because the fibers are oriented almost perpendicular to the image plane, which leads to a small retardation signal and a small image contrast. Therefore, the histogram is computed over the whole selected region, and the peak with lower (larger) transmittance is assumed to belong to fibers (surrounding tissue). The contrast values are computed from the respective peak values (numbers in pink and cyan) via $\mathcal{C}=(\max -\min ) /(\max +\min )$. The contrast for steep nerve fibers (coronal brain section) is much larger than for flat nerve fibers (sagittal and horizontal brain section).

\section{APPENDIX C: GENERATION OF ARTIFICIAL FIBER CONFIGURATIONS}

\section{Densely grown fiber bundle}

The bundle of densely grown fibers [see Fig. 3(b)(i)] is generated by in-house-developed software: $N=700$ circles with uniformly distributed diameters $(d \in[1.0,1.6] \mu \mathrm{m})$ are randomly uniformly placed in the $x y$ plane (in an area of $45 \times 30 \mu \mathrm{m}^{2}$ ). The circles are initialized with a random speed (maximum $0.1 \mu \mathrm{m}$ displacement per step) and collide with each other (assuming elastic collision with particle mass $r^{2}$ ) until a solution is reached without a collision in the $x y$ plane. To obtain well-distributed fibers, the previous step is repeated 250 times before the positions of the circles are stored. To obtain a 3D fiber volume, the circle positions are stored while incrementing the $z$ position by $1 \mu \mathrm{m}$ per step. To generate fiber bundles with different inclination angles, the resulting bundle of densely grown fibers is rotated around the $y$ axis with respect to the center position and cropped to a volume of $30 \times 30 \times 30 \mu \mathrm{m}^{3}$. To prevent fibers from touching each other after discretization, all fiber diameters are reduced by $5 \%$. In the resulting fiber bundle, about $60 \%$ of the volume is filled with fibers.

\section{Inhomogeneous fiber bundles}

Inhomogeneous fiber bundles, like the bundle with broad fiber orientation distribution [Fig. 3] or crossing fibers [Figs. 5(a) and 5(b)], are generated by in-house-developed software [86] which allows collision control in 3D. Starting from well-distributed straight fibers with $N=700$ and $d \in$ $[1.0,1.6] \mu \mathrm{m}$ (obtained after 250 steps as described in the previous section), the fibers are divided iteratively into segments of $2-5 \mu \mathrm{m}$ and assigned a random displacement in the $x, y$, and $z$ direction. The resulting fiber segments are split or merged until the length of each segment is again between 2 and $5 \mu \mathrm{m}$, ensuring that the maximum angle 
between adjacent segments is less than $20^{\circ}$. When a collision between two segments is detected, the segments are exposed to a small repelling force, and the previous step is repeated until no more collisions are detected. To prevent fibers from touching each other, all fiber diameters are reduced by $5 \%$. The resulting fiber bundle is cropped to a volume of $30 \times 30 \times 30 \mu \mathrm{m}^{3}$. The fiber bundles are generated from different configurations of straight fibers and different random displacements.

(i) Bundle with broad fiber orientation distribution [Fig. 3(b)(ii)].- The fiber bundle is generated from a bundle of straight horizontal fibers in the $x$ direction and a maximum random displacement of $10 \mu \mathrm{m}$. In the resulting fiber bundle, about $33 \%$ of the volume is filled with fibers. To generate fiber bundles with different inclination angles, the resulting bundle is rotated around the $y$ axis with respect to the center position.

(ii) Separate crossing fiber bundles [Fig. 5(a)].-The bundle of straight horizontal fibers in the $x$ direction is divided into an upper and a lower bundle of thickness $z / 2$, respectively. The upper bundle is rotated around the $z$ axis about the center position by an angle $+\chi / 2$, and the lower bundle is rotated by an angle $-\chi / 2$, resulting in two separate bundles with crossing angle $\chi$ [cf. Fig. 5(a)]. The resulting fibers are used as input for the algorithm with a maximum displacement of $1 \mu \mathrm{m}$. Depending on the crossing angle of the resulting fiber bundle, between $40 \%$ and $50 \%$ of the volume is filled with fibers.

(iii) Interwoven crossing fiber bundles [Fig. 5(a)].Each fiber layer in the $z$ direction of the straight horizontal fiber bundle (oriented in the $x$ direction) is rotated alternately by $\pm \chi / 2$ [cf. Fig. 5(b)]. The resulting fibers are used as input for the algorithm with a maximum displacement of $1 \mu \mathrm{m}$. Depending on the crossing angle of the resulting fiber bundles, between $40 \%$ and $50 \%$ of the volume is filled with fibers.

(iv) Mutually orthogonal, interwoven fiber bundles [Fig. 5(b)].-The straight horizontal fiber bundle (oriented in the $x$ direction) is divided into three types of alternating layers: One layer is rotated $+45^{\circ}$ around the $z$ axis, one $-45^{\circ}$ around the $z$ axis, and one $+90^{\circ}$ around the $y$ axis, yielding two horizontal fiber bundles in the $x y$ plane and one vertical fiber bundle oriented along the $z$ axis. The resulting fibers are used as input for the algorithm with a maximum displacement of $1 \mu \mathrm{m}$. In the resulting fiber bundle [cf. Fig. 5(c)], ca. $32 \%$ of the volume is filled with fibers.

\section{APPENDIX D: MODEL OF THE NERVE FIBERS}

Most axons in the white matter of the brain are surrounded by a myelin sheath, which consists of densely packed cell membranes [87,88]. Figure 15(c) shows the layered structure (a)

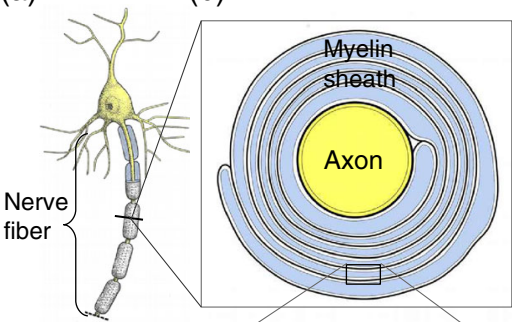

(c)

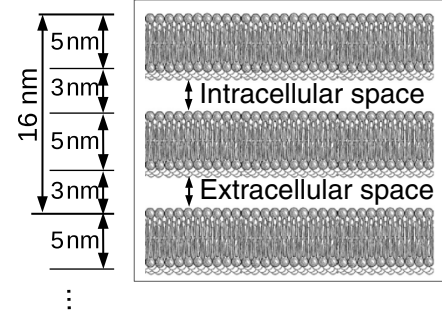

(e)

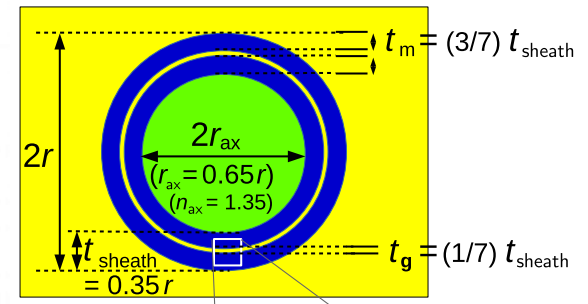

(d)

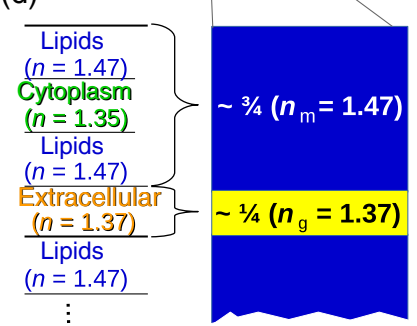

FIG. 15. Modeling of nerve fibers. (a) Schematic drawing of a nerve fiber (myelinated axon). (b) Cross section through the nerve fiber showing the inner axon and the surrounding myelin sheath (formed by a type of glial cell which spirally wraps around the axon). (c) Schematic representation of the myelin structure consisting of several lipid bilayers (5-nm-thick cell membranes) with an intracellular or cytoplasmic and an extracellular space of about $3 \mathrm{~nm}$. (d) Each cell layer (two lipid bilayers with separating cytoplasm) is considered as one "myelin layer" with an effective refractive index $n_{m}=1.47$ (blue), the extracellular space is considered to be filled with glycerin solution ("glycerin layer") with a refractive index $n_{g}=1.37$ (yellow). The myelin and glycerin layers are assumed to contribute $3 / 4$ and $1 / 4$ to the overall myelin sheath thickness $t_{\text {sheath }}$, respectively. (e) Nerve fibers are modeled with double myelin layers with thickness $t_{m}=(3 / 7) t_{\text {sheath }}$ and a single separating glycerin layer with thickness $t_{g}=(1 / 7) t_{\text {sheath. }}$. The myelin sheath thickness contributes approximately one-third to the overall fiber radius $\left(t_{\text {sheath }}=0.35 r\right)$. The inner axon is modeled with a radius $r_{\mathrm{ax}}=0.65 r$ and a refractive index $n_{\mathrm{ax}}=1.35$. 
of the myelin sheath: It consists of alternating layers of cell membranes (lipid bilayers of about $5 \mathrm{~nm}$ thickness) and intracellular or cytoplasmic and extracellular space (of about $3 \mathrm{~nm}$ thickness) $[39,89]$. As the extracellular membranes are not fused and swell in water $[39,87]$, it is assumed that the extracellular space is filled with the glycerin solution used for embedding the brain sections.

The refractive indices $n$ of the layers can be estimated from literature values of cell membranes or lipids $(n=1.47$ [90], neglecting any proteins), cytoplasm $(n=1.35$ [91]), and glycerin solution $(n=1.37$, measured with a digital refractometer).

For the simulation studies in Secs. III and IV, a simplified model is used to represent the myelin sheath [see Fig. 15(d)]: Each cell layer (two lipid bilayers with separating cytoplasm) is considered as one myelin layer with an effective refractive index $n_{m}=1.47$ (blue), and the extracellular space is considered to be filled with glycerin solution (glycerin layer) with a refractive index $n_{g}=1.37$ (yellow). Assuming that the extracellular space increases when being embedded in glycerin, the myelin and glycerin layers are assumed to contribute $3 / 4$ and $1 / 4$ to the overall myelin sheath thickness $t_{\text {sheath }}$, respectively. The refractive index of the cytoplasmic layer is neglected in this model.

The myelin sheath thickness contributes approximately one-third to the overall fiber radius $r$ [92]. Hence, the myelin sheath thickness is chosen to be $t_{\text {sheath }}=0.35 r$ and the radius of the inner axon $r_{\mathrm{ax}}=0.65 r$. The refractive index of the axon (green) is chosen to correspond to the refractive index of cytoplasm $\left(n_{\mathrm{ax}}=1.35\right)$. The myelin sheath is modeled as double myelin layers with thickness $t_{m}=(3 / 7) t_{\text {sheath }}$ each and a single glycerin layer with thickness $t_{g}=(1 / 7) t_{\text {sheath }}$ separating the myelin layers. Interruptions of the myelin sheath (nodes of Ranvier) and the small space between the axon and myelin sheath (periaxonal space [88]) are neglected in this model.

\section{APPENDIX E: FDTD ALGORITHM}

The propagation of the polarized light wave through the brain tissue samples (nerve fiber configurations) is simulated by a massively parallel 3D Maxwell solver based on a conditionally stable FDTD algorithm [45]. The algorithm computes the electromagnetic field components numerically by discretizing space and time and approximating Maxwell's curl equations by finite differences: The discretization is realized with a cubic Yee grid [93] (each electric field component is surrounded by four magnetic field components and vice versa) and a leapfrog time-stepping scheme. The spatial and temporal derivatives in Maxwell's curl equations are approximated by second-order central differences. For more details, see Menzel et al. [46].

The simulations are performed with the software TDME3D $^{\text {TM }}[47,68]$ - a massively parallel three-dimensional FDTD Maxwell solver, copyright European Marketing and Business Development BVBA. The software solves
Maxwell's equations for arbitrary-shaped objects that are illuminated by arbitrary incident plane waves and that consist of linear, isotropic, lossy materials with known permeability, permittivity, and conductivity. For the FDTD simulations, a combined algorithmic approach is used: In free space, Yee's algorithm is applied. To compute the interaction of the light with brain tissue, an unconditionally stable Lie-Trotter-Suzuki product formula approach is used. This approach results in a computationally efficient but conditionally stable algorithm. For more information, see De Raedt [94]. The simulations are performed on the supercomputer JUQUEEN [75] at Forschungszentrum Jülich GmbH, Germany.

\section{APPENDIX F: SIMULATION PARAMETERS}

Table II lists the parameters that are used for the simulation studies in Secs. III and IV.

All fiber configurations are generated in a volume of $30 \times 30 \times 30 \mu \mathrm{m}^{3}$. As described in Appendix D, each fiber is modeled by an inner axon and a surrounding myelin sheath with two layers and different refractive indices [see Fig. 15(e)]. The surrounding medium is assumed to be homogeneous with a refractive index $n_{\text {surr }}=$ $n_{g}=1.37$, which corresponds to the refractive index of gray brain matter as well as to the refractive index of the

TABLE II. Parameters for the simulation studies in Secs. III and IV: expenses of one simulation run (computation of one fiber configuration, one wavelength, and one angle of incidence on JUQUEEN), dimensions of the simulation volume, and fiber properties (radius $r$, thickness $t$, and refractive index $n$ ).

General simulation parameters

\begin{tabular}{|c|c|}
\hline Yee mesh size & $\Delta=25 \mathrm{~nm}$ \\
\hline Courant factor & $C=0.8$ \\
\hline Number of periods & 200 \\
\hline MPI grid & $16 \times 16 \times 16$ \\
\hline Core hours & About $7000-8000$ \\
\hline Wall time & About $1: 45-2: 00 \mathrm{~h}$ \\
\hline Minimum memory required & About $260-360 \mathrm{~GB}$ \\
\hline \multicolumn{2}{|l|}{ Simulation box } \\
\hline Volume & $x \times y \times z=30 \times 30 \times 35 \mu \mathrm{m}^{3}$ \\
\hline Boundaries & UPML $(1 \mu \mathrm{m}$ thick $)$ \\
\hline \multicolumn{2}{|l|}{ Surrounding medium } \\
\hline Dimensions & $x \times y \times z=30 \times 30 \times 31 \mu \mathrm{m}^{3}$ \\
\hline Refractive index & $n_{\text {surr }}=1.37$ \\
\hline \multicolumn{2}{|l|}{ Fiber configuration } \\
\hline Volume & $x \times y \times z=30 \times 30 \times 30 \mu \mathrm{m}^{3}$ \\
\hline Fiber radius & $r \sim 0.5 \mu \mathrm{m}$ \\
\hline Axon & $r_{\mathrm{ax}}=0.65 r, n_{\mathrm{ax}}=1.35$ \\
\hline Myelin sheath & $t_{\text {sheath }}=0.35 r=t_{m}+t_{g}+t_{m}$ \\
\hline Double myelin layers & $t_{m}=\frac{3}{7} t_{\text {sheath }}, n_{m}=1.47$ \\
\hline Single glycerin layer & $t_{g}=\frac{1}{7} t_{\text {sheath }}, n_{g}=1.37$ \\
\hline
\end{tabular}


surrounding glycerin solution. To account for the fact that the brain sections are embedded in glycerin solution (see Appendix A 1), 0.5- $\mu \mathrm{m}$-thick layers of glycerin solution (with refractive index $n_{g}=1.37$ ) are added at the bottom and on top of the sample, yielding a medium with dimensions $30 \times 30 \times 31 \mu \mathrm{m}^{3}$. The dimensions of the simulation box are chosen to be $30 \times 30 \times 35 \mu \mathrm{m}^{3}$ to leave some space for light source and detection planes. The simulation volume is surrounded by uniaxial perfectly matched layer (UPML) absorbing boundaries of $1 \mu \mathrm{m}$ thickness, thick enough to prevent light from being reflected back into the simulation volume. The different components of the sample are simulated as dielectrics with real refractive indices (as described in Appendix D). Absorption is neglected, because the absorption coefficients of brain tissue are small [50,51].

The simulation studies are performed for a duration of 200 periods and a Courant factor of 0.8 . The Yee mesh size is chosen to be $\Delta=25 \mathrm{~nm}$. This mesh size is just large enough to account for the double myelin layers of the nerve fiber model (see Appendix D: The glycerin layer for fibers with $1 \mu \mathrm{m}$ diameter is $25 \mathrm{~nm}$ thick).

The light source is modeled as a plane monochromatic wave. The simulation studies are performed for normally incident and coherent light with left-handed circular polarization and a wavelength of $550 \mathrm{~nm}$ (corresponding to the peak wavelength of the employed light source; see Appendix A 2). Using an MPI grid of $16 \times 16 \times 16$ on JUQUEEN, each simulation run (i.e., the calculation of one configuration, one wavelength, and one angle of incidence) consumes between 7000 and 8000 core hours, requires a minimum memory between 260 and $360 \mathrm{~GB}$, and lasts between 1:45 and 2:00 hours.

\section{APPENDIX G: COMPUTATION OF THE TRANSMITTED LIGHT INTENSITIES}

Figure 16 shows how the 3D-PLI measurement is modeled by means of FDTD simulations. For the simulations, a

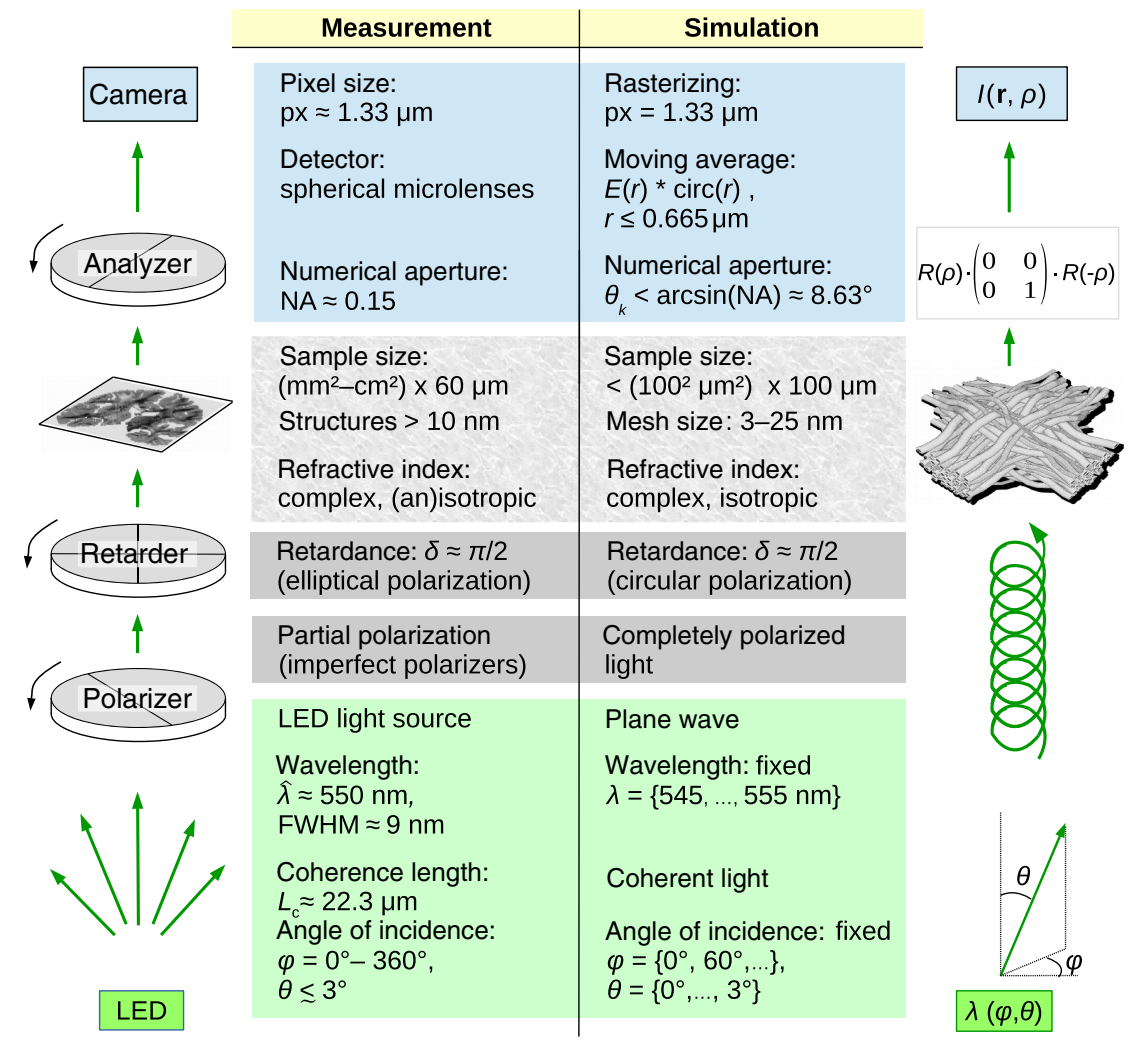

FIG. 16. Modeling of the 3D-PLI measurement. The figure and table on the left-hand side show the optical components of the polarimeter (the order of the polarizing filters is different than in the measurement, but the setup is mathematically equivalent): light source (green), polarizer and retarder (dark gray), sample (light gray), and objective lens, detector, and camera (blue). The table and figure on the right-hand side show how the optical elements are modeled by FDTD simulations: The incoherent and diffusive light source (LED) with peak wavelength $\hat{\lambda}$ and full width at half maximum (FWHM) is modeled by performing several simulation runs with plane waves that have different wavelengths $(\lambda)$ and angles of incidence $(\varphi, \theta)$. The modeled light source emits coherent light that is circularly polarized. The tissue sample is represented by an artificial fiber architecture, the rotating analyzer by a rotated Jones matrix [with rotation matrix $R(\rho)$ ]. The numerical aperture (NA) of the imaging system is modeled by considering only wave vector angles $\theta_{k}<\arcsin (\mathrm{NA})$. The spherical microlenses of the camera detector are modeled by performing a moving average over the area of the microlens with radius $r=1.33 \mu \mathrm{m} / 2$. 
mathematically equivalent polarimetric setup of the employed microscope is considered in which the sample is illuminated by (left-handed) circularly polarized light and analyzed by a rotating linear polarizer (analyzer).

The computation of the transmitted light intensities consists of several steps.

(i) Maxwell solver.-After passing the polarizing filters in front of the sample (see Fig. 16, left), the light wave is left-handed circularly polarized. The propagation of the light wave through the sample is computed by TDME3D as described in Appendix E. The resulting light wave is represented by a superposition of monochromatic plane waves with different wave vectors $\mathbf{k}$ and real amplitudes $\mathbf{E}_{0, k}$ :

$$
\begin{gathered}
\mathbf{E}_{k}(\mathbf{r}, t)=\mathbf{E}_{0, k} \cos (\mathbf{k} \cdot \mathbf{r}-\omega t+\phi) \\
\equiv \mathbf{A}_{k} \cos (\mathbf{k} \cdot \mathbf{r}-\omega t)-\mathbf{B}_{k} \sin (\mathbf{k} \cdot \mathbf{r}-\omega t),
\end{gathered}
$$

where $\mathbf{r}$ and $t$ are the spatial and temporal coordinates, respectively, $\omega$ is the angular frequency, $\phi$ is the phase, and $\mathbf{A}_{k}$ and $\mathbf{B}_{k}$ are defined as $\mathbf{A}_{k}=\mathbf{E}_{0, k} \cos \phi$ and $\mathbf{B}_{k}=\mathbf{E}_{0, k} \sin \phi$, respectively.

Note that every index $k$ denotes a different wave vector $\mathbf{k}$ and is not related to the wave number $k=2 \pi / \lambda$ (the wavelength of the transmitted light waves is the same as for the ingoing light wave).

(ii) Yee shift.-Before further processing, the electromagnetic field components are shifted in the $x, y, z$ direction to the middle of the corresponding Yee cell, respectively:

$$
\begin{array}{rlrl}
E_{k, x}(\mathbf{r}, t): y \mapsto y+\Delta y / 2, & z \mapsto z+\Delta z / 2, \\
E_{k, y}(\mathbf{r}, t): x \mapsto x+\Delta x / 2, & & & \mapsto \mapsto z+\Delta z / 2, \\
E_{k, z}(\mathbf{r}, t): x \mapsto x+\Delta x / 2, & y & \mapsto y+\Delta y / 2,
\end{array}
$$

where $\Delta x=\Delta y=\Delta z$ is the side length of the cubic Yee cell.

For each shift $\Delta j$ in the direction $j=\{x, y, z\}$, the vector components $A_{k, i}$ and $B_{k, i}$ are recomputed as follows:

$$
\begin{aligned}
& \check{A}_{k, i}=A_{k, i} \cos \left(k_{j} \Delta j\right)-B_{k, i} \sin \left(k_{j} \Delta j\right), \\
& \check{B}_{k, i}=A_{k, i} \sin \left(k_{j} \Delta j\right)+B_{k, i} \cos \left(k_{j} \Delta j\right) .
\end{aligned}
$$

After performing the shifts specified in Eqs. (G3)-(G5), the resulting field vector is given by

$\mathbf{E}_{k}^{\prime}(\mathbf{r}, t)=\mathbf{A}_{k}^{\prime} \cos (\mathbf{k} \cdot \mathbf{r}-\omega t)-\mathbf{B}_{k}^{\prime} \sin (\mathbf{k} \cdot \mathbf{r}-\omega t)$.

(iii) Scattering pattern.-To study how much light is scattered under a certain angle (wave vector $\mathbf{k}$ ), the scattering pattern is computed, i.e., the intensity per wave vector normalized by the ingoing light intensity $\left(I_{0}\right)$ per image pixel (px):

$$
I_{k} \equiv \frac{\left|\mathbf{E}_{0, k}^{\prime}\right|^{2}}{I_{0} /(\# \mathrm{px})}=\frac{\left|\mathbf{A}_{k}^{\prime}\right|^{2}+\left|\mathbf{B}_{k}^{\prime}\right|^{2}}{I_{0} /(\# \mathrm{px})} .
$$

(iv) Rotating analyzer.-To model the 3D-PLI measurement, the electric field vector $\mathbf{E}_{k}^{\prime}(\mathbf{r}, t)$ is processed through the second linear polarizer (analyzer) rotated by angles $\rho$, yielding

$$
\begin{aligned}
\tilde{\mathbf{E}}_{k}(\mathbf{r}, t, \rho)= & \tilde{\mathbf{A}}_{k}(\rho) \cos (\mathbf{k} \cdot \mathbf{r}-\omega t) \\
& -\tilde{\mathbf{B}}_{k}(\rho) \sin (\mathbf{k} \cdot \mathbf{r}-\omega t)
\end{aligned}
$$

The $x$ and $y$ components of $\tilde{\mathbf{E}}_{k}(\mathbf{r}, t, \rho)$ are computed by multiplying $\mathbf{E}_{k}^{\prime}(\mathbf{r}, t)$ with the Jones matrix of a rotated linear polarizer $[95,96]$ :

$$
\begin{aligned}
\left(\begin{array}{c}
\tilde{E}_{k, x}(\mathbf{r}, t, \rho) \\
\tilde{E}_{k, y}(\mathbf{r}, t, \rho)
\end{array}\right) & =\left(\begin{array}{cc}
\cos \rho & -\sin \rho \\
\sin \rho & \cos \rho
\end{array}\right)\left(\begin{array}{ll}
0 & 0 \\
0 & 1
\end{array}\right)\left(\begin{array}{cc}
\cos \rho & \sin \rho \\
-\sin \rho & \cos \rho
\end{array}\right)\left(\begin{array}{c}
E_{k, x}^{\prime}(\mathbf{r}, t) \\
E_{k, y}^{\prime}(\mathbf{r}, t)
\end{array}\right) \\
& =\left(\begin{array}{c}
\sin \rho\left[E_{k, x}^{\prime}(\mathbf{r}, t) \sin \rho-E_{k, y}^{\prime}(\mathbf{r}, t) \cos \rho\right] \\
-\cos \rho\left[E_{k, x}^{\prime}(\mathbf{r}, t) \sin \rho-E_{k, y}^{\prime}(\mathbf{r}, t) \cos \rho\right]
\end{array}\right) .
\end{aligned}
$$

The $z$ component is computed by applying Maxwell's equation in free space and assuming $\tilde{\mathbf{E}}_{k}(\mathbf{r}, t, \rho)=\tilde{\mathbf{E}}_{0, k}(\rho) e^{i(\mathbf{k} \cdot \mathbf{r}-\omega t+\phi)}$ (plane monochromatic wave):

$$
\begin{aligned}
& \operatorname{div} \tilde{\mathbf{E}}_{k}(\mathbf{r}, t, \rho)=0 \Leftrightarrow \mathbf{k} \cdot \tilde{\mathbf{E}}_{k}(\mathbf{r}, t, \rho)=0 \\
& \Leftrightarrow \tilde{E}_{k, z}(\mathbf{r}, t, \rho)=-\frac{1}{k_{z}}\left[k_{x} \tilde{E}_{k, x}(\mathbf{r}, t, \rho)+k_{y} \tilde{E}_{k, y}(\mathbf{r}, t, \rho)\right] \\
& \stackrel{(G 11)}{=}-\frac{k_{x} \sin \rho-k_{y} \cos \rho}{k_{z}}\left[E_{k, x}^{\prime}(\mathbf{r}, t) \sin \rho-E_{k, y}^{\prime}(\mathbf{r}, t) \cos \rho\right] .
\end{aligned}
$$


(v) Objective lens.-The objective lens is assumed to be ideal, and both the specimen and detector are assumed to lie within the corresponding focal planes of the lens. Thus, the propagation of the electromagnetic wave between the sample and detector is assumed to be free, and $\tilde{\mathbf{E}}_{k}(\mathbf{r}, t, \rho)$ is evaluated at the $z$ position of the detection plane behind the sample (defined as $z=0$ ):

$$
\mathbf{r}=\left(r_{x}, r_{y}, 0\right)^{T} .
$$

To account for the numerical aperture (NA) of the objective lens, only $k$ vectors are processed that fulfill

$$
\theta_{k}=\arccos \left(\frac{k_{z}}{\sqrt{k_{x}^{2}+k_{y}^{2}+k_{z}^{2}}}\right) \leq \arcsin (\mathrm{NA}) .
$$

The employed imaging system has a numerical aperture of about 0.15 , so only $k$ vectors with angles $\theta_{k} \leq 8.6^{\circ}$ are used for processing.

(vi) Detector microlenses. - The camera sensor contains an array of spherical microlenses which bundle the light onto subjacent photodiodes for each image pixel. Assuming perfect microlenses and photodiodes that are completely covered by one microlens, respectively, the microlenses are modeled by applying a moving average over the area of the microlens. Instead of taking the magnification and the physical size of the microlenses into account, the microlenses are modeled with a diameter of $2 r_{0}=1.33 \mu \mathrm{m}$, corresponding to the pixel size of the microscope in object space:

$$
\begin{aligned}
\breve{\mathbf{E}}_{k}(r, t, \rho) & =\tilde{\mathbf{E}}_{k}(\mathbf{r}, t, \rho) * \operatorname{circ}(r), \\
\operatorname{circ}(r) & =\left\{\begin{array}{ll}
\frac{1}{\pi r_{0}^{2}}, & r<r_{0} \\
0, & r \geq r_{0}
\end{array} .\right.
\end{aligned}
$$

To obtain the full image information (independent of the detector pixel position), no rasterizing is applied.

(vii) Intensity.-In principle, the intensity detected by the camera sensor depends on the angle of incidence of the incident light: $I \cos \theta_{k}$. As the numerical aperture is sufficiently small (NA $=\sin \theta_{k} \approx 0.15 \Leftrightarrow \cos \theta_{k}>$ 0.9886 ), the angle dependence is neglected, which enables us to represent the intensity $I(\mathbf{r}, \rho)$ as a Fourier series in $\rho$, as described below.

With this assumption, the light intensity recorded by the camera is given by the absolute squared value of the electric field vector. To compute the intensity at a certain point $r$ in the image plane, the electric field vectors are summed over $\mathbf{k}$ and averaged over time:

$$
\begin{aligned}
I(\mathbf{r}, \rho) & \propto|\mathbf{E}(\mathbf{r}, \rho)|^{2} \equiv \frac{1}{T} \int_{0}^{T}\left|\sum_{\mathbf{k}} \breve{\mathbf{E}}_{k}(\mathbf{r}, t, \rho)\right|^{2} d t \\
& \propto\left|\mathrm{FT}^{-1}\left\{\tilde{\mathbf{A}}_{k}(\rho)+i \tilde{\mathbf{B}}_{k}(\rho)\right\} * \operatorname{circ}(r)\right|^{2},
\end{aligned}
$$

where $\mathrm{FT}^{-1}$ denotes the inverse discrete Fourier transform:

$$
\mathrm{FT}^{-1}\{f\}=\sum_{\mathbf{k}} f_{\mathbf{k}} e^{i \mathbf{k} \cdot \mathbf{r}} .
$$

The discrete Fourier transform (FT) is defined analogously.

To save computing time, the convolution in Eq. (G16) is replaced by a multiplication, making use of the convolution theorem:

$$
\begin{aligned}
I(\mathbf{r}, \rho) & \propto\left|\mathrm{FT}^{-1}\left\{\left[\tilde{\mathbf{A}}_{k}(\rho)+i \tilde{\mathbf{B}}_{k}(\rho)\right] \mathrm{FT}\{\operatorname{circ}(r)\}\right\}\right|^{2} \\
& =\left|\mathrm{FT}^{-1}\left\{\left[\tilde{\mathbf{A}}_{k}(\rho)+i \tilde{\mathbf{B}}_{k}(\rho)\right] 2 \frac{J_{1}\left(r_{0} k_{x y}\right)}{r_{0} k_{x y}}\right\}\right|^{2},
\end{aligned}
$$

where the function $J_{1}(x)$ is the Bessel function of the first kind of order one, with $k_{x y} \equiv \sqrt{k_{x}^{2}+k_{y}^{2}}$ and $r_{0}=0.665 \mu \mathrm{m}$.

To simplify notation, the following abbreviations are defined:

$$
\begin{gathered}
\tilde{\mathbf{E}}_{k}(\rho) \equiv\left[\tilde{\mathbf{A}}_{k}(\rho)+i \tilde{\mathbf{B}}_{k}(\rho)\right] 2 \frac{J_{1}\left(r_{0} k_{x y}\right)}{r_{0} k_{x y}}, \\
\mathbf{E}_{k}^{\prime} \equiv\left(\mathbf{A}_{k}^{\prime}+i \mathbf{B}_{k}^{\prime}\right) 2 \frac{J_{1}\left(r_{0} k_{x y}\right)}{r_{0} k_{x y}}, \\
\tilde{\mathbf{E}}(r, \rho) \equiv \mathrm{FT}^{-1}\left\{\tilde{\mathbf{E}}_{k}(\rho)\right\}, \\
\mathbf{E}^{\prime}(\mathbf{r}) \equiv \mathrm{FT}^{-1}\left\{\mathbf{E}_{k}^{\prime}\right\} .
\end{gathered}
$$

The intensity is then given by

$$
I(\mathbf{r}, \rho) \propto\left|\tilde{\mathcal{E}}_{x}(\mathbf{r}, \rho)\right|^{2}+\left|\tilde{\mathcal{E}}_{y}(\mathbf{r}, \rho)\right|^{2}+\left|\tilde{\mathcal{E}}_{z}(\mathbf{r}, \rho)\right|^{2} .
$$

The $x$ and $y$ components of the electric field vector $\tilde{\mathbf{E}}_{k}(\mathbf{r}, t, \rho)$ behind the rotating analyzer are computed from $\mathbf{E}_{k}^{\prime}(\mathbf{r}, t)=\mathbf{A}_{k}^{\prime} \cos (\mathbf{k} \cdot \mathbf{r}-\omega t)-\mathbf{B}_{k}^{\prime} \sin (\mathbf{k} \cdot \mathbf{r}-\omega t)$ according to Eq. (G11). As the equation is linear in the $x$ and $y$ components of $\mathbf{E}_{k}^{\prime}(\mathbf{r}, t)$, the $x$ and $y$ components of $\left\{\mathbf{A}_{k}^{\prime}, \mathbf{B}_{k}^{\prime}, \mathbf{E}^{\prime}{ }_{k}\right\}$ are transformed to $\left\{\tilde{\mathbf{A}}_{k}(\rho), \tilde{\mathbf{B}}_{k}(\rho), \tilde{\mathbf{E}}_{k}(\rho)\right\}$ according to the same equation. As the Fourier transform is independent from $\rho$, Eq. (G11) also holds for the $x$ and $y$ components of $\mathbf{E}^{\prime}(\mathbf{r})$ and $\tilde{\mathbf{E}}(\mathbf{r}, \rho)$, yielding Fourier coefficients of the order of zero and two: 


$$
\begin{aligned}
& \left|\tilde{\mathcal{E}}_{x}(\mathbf{r}, \rho)\right|^{2}+\left|\tilde{\mathcal{E}}_{y}(\mathbf{r}, \rho)\right|^{2} \stackrel{(G 11)}{=} \sin ^{2} \rho\left|\mathcal{E}_{x}^{\prime}(\mathbf{r})\right|^{2}+\cos ^{2} \rho\left|\mathcal{E}_{y}^{\prime}(\mathbf{r})\right|^{2}-\sin \rho \cos \rho\left[\mathcal{E}_{x}^{\prime}(\mathbf{r}) \mathcal{E}_{y}^{\prime *}(\mathbf{r})+\mathcal{E}_{x}^{\prime *}(\mathbf{r}) \mathcal{E}_{y}^{\prime}(\mathbf{r})\right] \\
& =\underbrace{\frac{1}{2}\left[\left|\mathcal{E}_{x}^{\prime}(\mathbf{r})\right|^{2}+\left|\mathcal{E}_{y}^{\prime}(\mathbf{r})\right|^{2}\right]}_{c_{o}}+\underbrace{\frac{1}{2}\left[\left|\mathcal{E}_{y}^{\prime}(\mathbf{r})\right|^{2}-\left|\mathcal{E}_{x}^{\prime}(\mathbf{r})\right|^{2}\right]}_{c_{2}} \cos (2 \rho) \underbrace{-\frac{1}{2}\left[\mathcal{E}_{x}^{\prime}(\mathbf{r}) \mathcal{E}_{y}^{*}(\mathbf{r})+\mathcal{E}_{x}^{* *}(\mathbf{r}) \mathcal{E}_{y}^{\prime}(\mathbf{r})\right]}_{d_{2}} \sin (2 \rho)
\end{aligned}
$$

$$
\equiv c_{0}(\mathbf{r})+c_{2}(\mathbf{r}) \cos (2 \rho)+d_{2}(\mathbf{r}) \sin (2 \rho)
$$

where trigonometric identifies have been used: $\left[\cos ^{2} x=\frac{1}{2}+\frac{1}{2} \cos (2 x), \sin x \cos x=\frac{1}{2} \sin (2 x)\right]$.

Similar analytical calculations yield Fourier coefficients of the orders of zero, two, and four:

$$
\begin{aligned}
\left|\tilde{\mathcal{E}}_{z}(\mathbf{r}, \rho)\right|^{2}= & e_{0}(\mathbf{r})+e_{2}(\mathbf{r}) \cos (2 \rho)+f_{2}(\mathbf{r}) \sin (2 \rho) \\
& +e_{4}(\mathbf{r}) \cos (4 \rho)+f_{4}(\mathbf{r}) \sin (4 \rho),
\end{aligned}
$$

where $e_{m}(\mathbf{r})$ and $f_{m}(\mathbf{r})$ are functions of the inverse discrete Fourier transforms:

$$
\begin{array}{ll}
X_{x}(\mathbf{r}) \equiv \mathrm{FT}^{-1}\left\{\frac{k_{x}}{k_{z}} \mathcal{E}_{k, x}^{\prime}\right\}, & X_{y}(\mathbf{r}) \equiv \mathrm{FT}^{-1}\left\{\frac{k_{y}}{k_{z}} \mathcal{E}_{k, x}^{\prime}\right\}, \\
Y_{x}(\mathbf{r}) \equiv \mathrm{FT}^{-1}\left\{\frac{k_{x}}{k_{z}} \mathcal{E}_{k, y}^{\prime}\right\}, & Y_{y}(\mathbf{r}) \equiv \mathrm{FT}^{-1}\left\{\frac{k_{y}}{k_{z}} \mathcal{E}_{k, y}^{\prime}\right\} .
\end{array}
$$

Thus, the transmitted light intensity $I(\mathbf{r}, \rho)$ can be written in terms of a Fourier series:

$$
\begin{aligned}
I(\mathbf{r}, \rho) \propto & \left|\tilde{\mathcal{E}}_{x}(\mathbf{r}, \rho)\right|^{2}+\left|\tilde{\mathcal{E}}_{y}(\mathbf{r}, \rho)\right|^{2}+\left|\tilde{\mathcal{E}}_{z}(\mathbf{r}, \rho)\right|^{2} \\
= & a_{0}(\mathbf{r})+a_{2}(\mathbf{r}) \cos (2 \rho)+b_{2}(\mathbf{r}) \sin (2 \rho) \\
& +a_{4}(\mathbf{r}) \cos (4 \rho)+b_{4}(\mathbf{r}) \sin (4 \rho), \\
a_{0}(\mathbf{r}) \equiv & c_{0}(\mathbf{r})+e_{0}(\mathbf{r}), \quad a_{2}(\mathbf{r}) \equiv c_{2}(\mathbf{r})+e_{2}(\mathbf{r}), \\
b_{2}(\mathbf{r}) \equiv & d_{2}(\mathbf{r})+f_{2}(\mathbf{r}), \quad a_{4}(\mathbf{r}) \equiv e_{4}(\mathbf{r}), \\
b_{4}(\mathbf{r}) \equiv & f_{4}(\mathbf{r}),
\end{aligned}
$$

where the Fourier coefficients $a_{m}(\mathbf{r})$ and $b_{m}(\mathbf{r})$ are computed from the six inverse discrete Fourier transforms defined above: $\mathcal{E}_{x}^{\prime}(\mathbf{r}), \quad \mathcal{E}_{y}^{\prime}(\mathbf{r}), \quad X_{x}(\mathbf{r}), \quad X_{y}(\mathbf{r}), \quad Y_{x}(\mathbf{r})$, and $Y_{y}(\mathbf{r})$.

For non-normally incident light $\left(k_{x} \neq 0\right.$ or $\left.k_{y} \neq 0\right)$, the transmitted light intensity contains Fourier coefficients of the order of four [cf. Eq. (G27)].

Using Eq. (G30), the light intensity is computed for arbitrary rotation angles $\rho$ and normalized by the ingoing light intensity per image pixel:

$$
I_{N}(\mathbf{r}, \rho)=\frac{I(\mathbf{r}, \rho)}{I_{0} /(\# \mathrm{px})}
$$

In the experiment, the measured light intensities are normalized by the light intensities measured without a specimen to compensate for filter inhomogeneities. This image calibration could be modeled by performing an additional simulation run without a sample. To save computing time, the simulated light intensities are simply normalized by $I_{0}$ (without considering the imaging system), and only relative values are used for the comparison between measured and simulated light intensities.

The Fourier coefficient of the order of zero $a_{0, N}(\mathbf{r})$ obtained from the simulated normalized transmitted light intensity $I_{N}(\mathbf{r}, \rho)$ is used to compute the simulated transmittance images $I_{T, N}(\mathbf{r})$ :

$$
I_{T, N}(\mathbf{r}) \equiv a_{0, N}(\mathbf{r}) .
$$

Figure S5 in Supplemental Material [55] summarizes the most important steps of computing the transmitted light intensities for 3D-PLI simulations. The computation is carried out in PYTHON (version 2.7.6) using the NumPy package (version 1.12.1) [97,98]. To obtain the intensity at a certain pixel position $(x, y)$, the inverse discrete Fourier transform is computed in two dimensions by means of the fast Fourier transform (FFT) [99]. To enable an efficient use of the FFT, the number of grid points in $x$ and $y\left(N_{x}\right.$ and $\left.N_{y}\right)$ are set to be a multiple of two:

$$
\begin{aligned}
& N_{x}^{\prime}=2^{m_{x}}>N_{x}, \\
& N_{y}^{\prime}=2^{m_{y}}>N_{y} .
\end{aligned}
$$

\section{APPENDIX H: ERROR ESTIMATION OF SIMULATION RESULTS}

When modeling the optical components of the imaging system, the limitations of the simulation software need to be taken into account: The simulated light wave is completely polarized and coherent, the materials are characterized by isotropic refractive indices, and the size and resolution of the simulated geometries are limited due to finite computing time.

Using completely polarized light for the simulations implies that the optical elements are assumed to be ideal 
(unpolarized light source, ideal polarizing filters, no polarization sensitivity of the camera). For the employed polarizing microscope, these assumptions are reasonable, because the optical components are of high quality. Moreover, the transmittance can be considered to be mostly independent from the polarization properties of the imaging system.

The simulation studies in Sec. III are performed for a reduced sample size $\left(30 \times 30 \times 30 \mu \mathrm{m}^{3}\right)$ and 200 periods. Simulations with larger sample sizes in $x$ or $y$ and more periods yield similar results [67].

To further reduce computing time, the simulation studies are performed for a simplified nerve fiber model [axon surrounded by double myelin layers; cf. Fig. 15(e)], a Yee mesh size of $25 \mathrm{~nm}$, and normally incident light with $550 \mathrm{~nm}$ wavelength. To estimate the accuracy of the simulation results, the transmittance images are simulated for different numbers of myelin layers $L$, different Yee mesh sizes $\Delta$, different wavelengths $\lambda$, and different angles of incidence $\theta$. To study the influence of one simulation parameter at once, only one simulation parameter is varied while all other simulation parameters are chosen as in Table II (with normally incident light and $550 \mathrm{~nm}$ wavelength).

To estimate the accuracy of the resulting transmittance images, the absolute relative difference between the mean values (ARDM) and the relative mean absolute difference $(R M A D)$ between the images are computed:

$$
\begin{aligned}
& \mathrm{ARDM} \equiv\left|\frac{\langle\text { image }\rangle-\langle\text { ref image }\rangle}{\langle\text { ref image }\rangle}\right|, \\
& \mathrm{RMAD} \equiv \frac{\langle| \text { image }- \text { ref image }|\rangle}{\mid\langle\text { ref image }\rangle \mid} .
\end{aligned}
$$

In this notation, the "image" refers to the transmittance image for which the absolute relative difference is computed (obtained, e.g., from simulations with different Yee mesh sizes). The "reference image" is the transmittance image used for comparison (obtained, e.g., from the simulation with minimum mesh size). The symbol \langle\rangle represents the average over all image pixels. As the simulation studies mostly investigate the mean transmittance values, the ARDM is a direct measure for the accuracy of the simulation results, while the RMAD is a measure for the reliability of the ARDM as an error estimate.

\section{Different numbers of myelin layers}

To estimate the accuracy of the simplified nerve fiber model, a straight single fiber with reduced simulation volume [see Fig. 17(a)] is simulated for different numbers $L$ of myelin layers with thickness $t_{m}$ (and $L-1$ separating glycerin layers with thickness $t_{g}=t_{m} / 3$ ) as well as for a realistic model of the myelin sheath consisting of 43 thin layers [see Fig. 17(b), right]. The Yee mesh size is chosen to be small enough to resolve all geometric features: For most samples, the mesh size is chosen to be one-third of the glycerin layer thickness $\left(\Delta=t_{g} / 3\right)$. Fibers with two myelin layers $(L=2)$ are also simulated for larger mesh sizes $\left(\Delta=t_{g} / 2=12.5 \mathrm{~nm}\right.$ and $\left.\Delta=t_{g}=25 \mathrm{~nm}\right)$. The realistic myelin sheath is simulated for $\Delta=t_{g}=3 \mathrm{~nm}$, consuming 288358 core hours on JUQUEEN (using an MPI grid of $64 \times 64 \times 16$ ).

Figure 17(c) shows the corresponding transmittance images, mean values, and line profiles obtained from 3D-PLI simulations with normally incident light and $\lambda=$ $550 \mathrm{~nm}$ for the straight single fibers shown in Fig. 17(b). The mean values and line profiles for $L \geq 1$ look similar. For better comparison, Fig. 17(d) shows the absolute relative differences (ARDM and RMAD) between the transmittance images with $L=\{0,1,2,3,4,5\}$ and the transmittance image with a realistic myelin sheath. The relative differences decrease with an increasing number of myelin layers $L$ and with decreasing mesh size $\Delta$. A fiber with two or more myelin layers and a mesh size $\Delta=t_{g} / 3$ yields similar transmittance values as the fiber with a realistic myelin sheath. With an increasing mesh size, the relative differences increase. For a fiber with double myelin layers and a mesh size $\Delta=12.5 \mathrm{~nm}(25 \mathrm{~nm})$, the differences are ARDM $\approx 1.2 \%(2.3 \%)$ and $\mathrm{RMAD} \approx 1.6 \%$ (2.8\%). For a mesh size of $25 \mathrm{~nm}$, the differences are still smaller than for a fiber without or with a single myelin layer. Thus, a fiber with double myelin layers and a mesh size of $25 \mathrm{~nm}$ is a good compromise between accuracy and computing time and is used for all simulation studies in Secs. III and IV. In interesting cases, the simulations are repeated for a reduced mesh size $(\Delta=12.5 \mathrm{~nm})$; see the black crosses in Fig. 18(b).

\section{Different wavelengths, angles of incidence, and Yee mesh sizes}

The light source of the employed polarizing microscope emits light with slightly different wavelengths $[\lambda=(550 \pm 5) \mathrm{nm}]$ and different angles of incidence (the sample is illuminated under angles $\theta<3^{\circ}$ ) [67]. To model this incoherent and diffusive light source, several simulation runs with different wavelengths $\lambda$ and angles of incidence $(\varphi, \theta)$ are performed, and the resulting intensities are added incoherently. A comparison of simulated and experimental data for a well-defined sample (USAF-1951 resolution target) reveal that the light source can sufficiently be modeled by three different wavelengths $(\lambda=\{545,550,555\} \mathrm{nm})$ weighted according to the wavelength spectrum and five angles of incidence $\left(\theta=0^{\circ}\right.$; $\theta=3^{\circ}, \varphi=\left\{0^{\circ}, 90^{\circ}, 180^{\circ}, 270^{\circ}\right\}$ ) [67].

The simulation studies in Secs. III and IV are performed only for normally incident light and a single wavelength $(\lambda=550 \mathrm{~nm})$. To estimate the accuracy of the simulation results, especially for the transmittance curves in Fig. 3(b), 
(a)
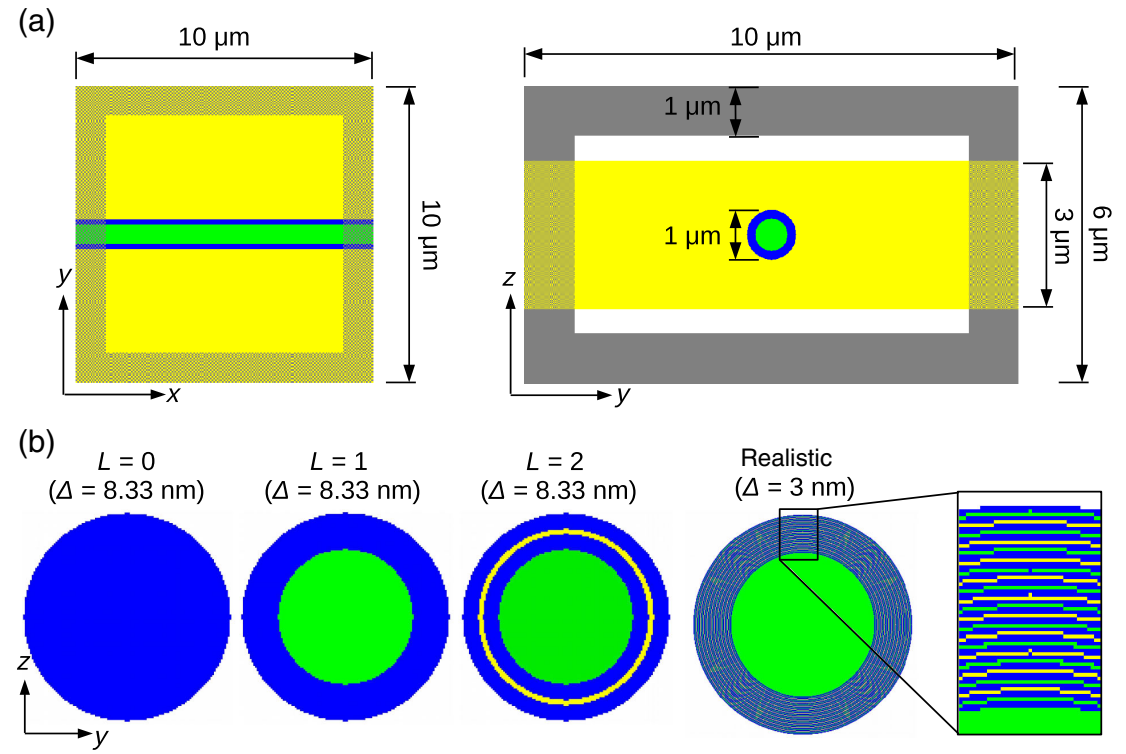

(c) $L=0$

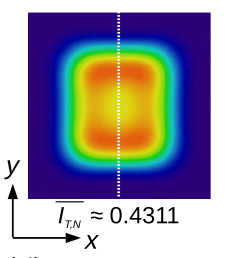

$L=1$
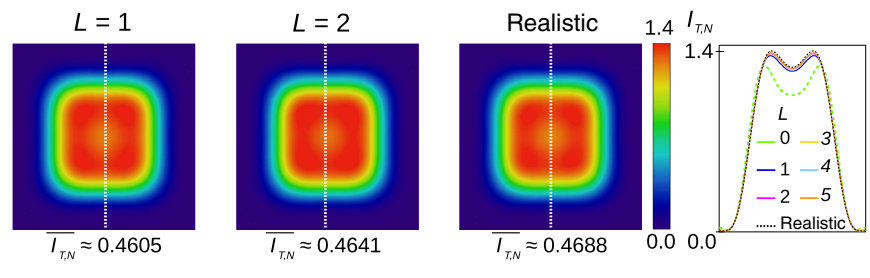

(d)
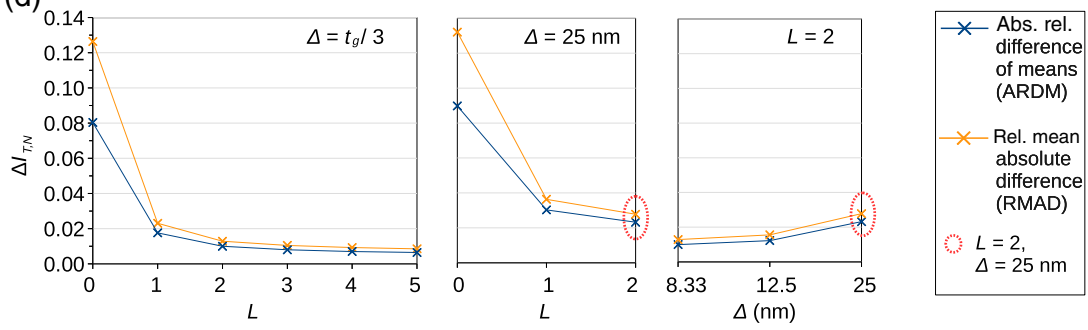

FIG. 17. Error estimation for different numbers of myelin layers. (a) Dimensions of the simulation volume ( $x y$ or $y z$ plane) used to simulate a straight single fiber with different numbers of myelin layers. (b) Cross section through fibers with different numbers $L$ of myelin layers and different Yee mesh sizes $\Delta$. All fibers are modeled with a diameter of $1 \mu \mathrm{m}$, consisting of an inner axon (green) with a diameter of $0.65 \mu \mathrm{m}$ and a surrounding myelin sheath with a thickness of $0.175 \mu \mathrm{m}$. The myelin sheath is composed of alternating layers of myelin (blue) and glycerin (yellow), and the myelin layers are 3 times thicker than the glycerin layers. The realistic model of the myelin sheath contains 22 layers of 5-nm-thick cell membranes (blue), interrupted by 3-nm-thick alternating layers of cytoplasm (green) and surrounding glycerin solution (yellow), yielding a myelin sheath composed of 43 thin layers. The refractive indices are 1.35 for the axon or cytoplasm (green), 1.37 for the glycerin solution (yellow), and 1.47 for the myelin layers (blue). A motivation of the myelin sheath model and the corresponding refractive indices is shown in Fig. 15. (c) Normalized transmittance images, corresponding mean values $\overline{I_{T, N}}$, and transmittance profiles on the right (middle image pixels evaluated along the $y$ axis; see the white dashed lines) obtained from 3D-PLI simulations with different numbers $L$ of myelin layers and Yee mesh sizes defined in (b). The simulations are performed for normally incident light with $550 \mathrm{~nm}$ wavelength and simulation parameters specified in Table II. The profiles with nonitalic labels belong to the displayed transmittance images. (d) Relative differences between the transmittance images with different numbers $L$ of myelin layers and different mesh sizes $\Delta$ (relative to the glycerin layer thickness $t_{g}$ ) and the transmittance image with a realistic myelin sheath. The values for ARDM (blue) and RMAD (orange) are computed using Eqs. (H1) and (H2); the values surrounded in red belong to fibers with double myelin layers $(L=2)$ and $25 \mathrm{~nm}$ mesh size, which are used for the simulation studies in Secs. III and IV.

the 3D-PLI simulations for the bundle of densely grown fibers with inclination angles $\alpha=\left\{0^{\circ}, 10^{\circ}, \ldots, 90^{\circ}\right\}$ are performed for the three different wavelengths and five angles of incidence defined above. The resulting transmittance curves for $\mathrm{NA}=0.15$ (solid curves) and $\mathrm{NA}=1$ (dashed curves) are shown in Fig. 18(b). The simulations are performed for a Yee mesh size of $25 \mathrm{~nm}$. For some inclination angles, the simulations for $\mathrm{NA}=0.15$ are repeated for a smaller mesh size $(\Delta=12.5 \mathrm{~nm})$; see the black crosses. 


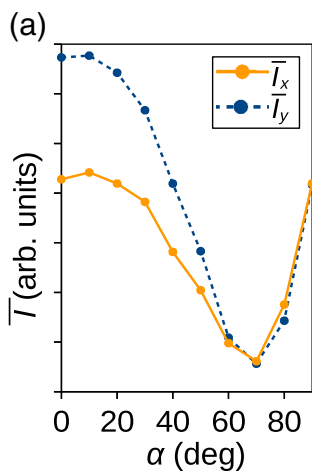

(b)

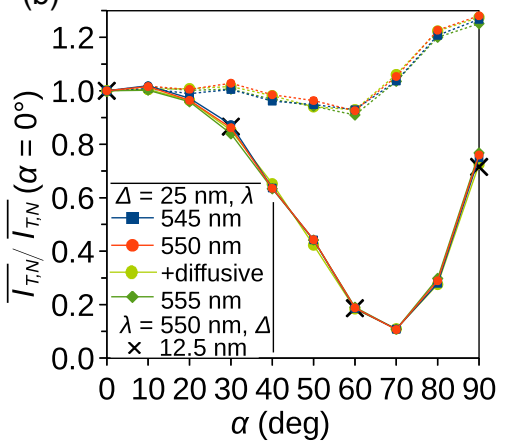

FIG. 18. Simulated transmitted light intensity for different simulation parameters. Bundle of densely grown fibers [cf. Fig. 3(a)] simulated for different inclination angles $\alpha$ : (a) Mean transmitted light intensity for light polarized along the $x$ axis $\left(\overline{I_{x}}\right)$ or along the $y$ axis $\left(\overline{I_{y}}\right)$ plotted against $\alpha$. The simulations are performed for a numerical aperture NA $=0.15$ using a normally incident plane wave with $550 \mathrm{~nm}$ wavelength and simulation parameters specified in Table II. (b) Transmittance curves (mean transmittance value $\overline{I_{T, N}}$ plotted against $\alpha$ ) obtained from 3D-PLI simulations for normally incident light with different wavelengths $\lambda=\{545,550,555\} \mathrm{nm}$ and a Yee mesh size of $\Delta=25 \mathrm{~nm}$. The simulations for $\lambda=550 \mathrm{~nm}$ are also performed for diffusive light (with angles of incidence $\left\{\theta=0^{\circ}\right\},\left\{\theta=3^{\circ} ; \varphi=4 \times 90^{\circ}\right\}$ ). The curves are normalized by the mean transmittance value of the horizontal bundle, respectively. The solid curves are computed for the numerical aperture of the imaging system $(\mathrm{NA}=0.15)$, and the dashed curves are computed for NA $=1$. The black crosses belong to simulations with $\mathrm{NA}=0.15, \lambda=550 \mathrm{~nm}$, and $\Delta=12.5 \mathrm{~nm}$.

The transmittance curves for different wavelengths and for diffusive light (obtained from simulation runs with different angles of incidence) look all very similar. The maximum difference between the normalized transmittance values is less than 0.03 . In addition, the simulations with a smaller mesh size (black crosses) yield similar results as the simulations with a larger mesh size (curves); the maximum difference between the normalized transmittance values is only about 0.005 . Thus, the transmittance curves for the bundle of densely grown fibers are not sensitive to small changes in the wavelength, angle of incidence, or mesh size, which demonstrates the validity of the simulation results.

[1] S. Herculano-Houzel, The Human Brain in Numbers: A Linearly Scaled-Up Primate Brain, Front. Hum. Neurosci. 3, 1 (2009).

[2] A. Longstaff, Neuroscience, 3rd ed., edited by E. Owen, BIOS Instant Notes (Garland Science, Taylor \& Francis, London, 2011).

[3] Y. Shi and A. W. Toga, Connectome Imaging for Mapping Human Brain Pathways, Mol. Psychiatry 22, 1230 (2017).
[4] S. Mori and J. Zhang, Principles of Diffusion Tensor Imaging and Its Applications to Basic Neuroscience Research, Neuron 51, 527 (2006).

[5] D. S. Tuch, T. G. Reese, M. R. Wiegell, and V. J. Wedeen, Diffusion MRI of Complex Neural Architecture, Neuron 40, 885 (2003).

[6] P. Hagman, J. P. Thiran, L. Jonasson, P. Vandergheynst, S. Clarke, P. Maeder, and R. Meuli, DTI Mapping of Human Brain Connectivity: Statistical Fibre Tracking, NeuroImage 19, 545 (2003).

[7] H.-C. Chang, M. Sundman, L. Petit, S. Guhaniyogi, M.-L. Chu, C. Petty, A. W. Song, and N. Chen, Human Brain Diffusion Tensor Imaging at Submillimeter Isotropic Resolution on a 3 Tesla Clinical MRI Scanner, NeuroImage 118, 667 (2015).

[8] A. Roebroeck, K. Miller, and M. Aggarwal, Ex Vivo Diffusion MRI of the Human Brain: Technical Challenges and Recent Advances, NMR Biomed. 32, e3941 (2018).

[9] E. Calabrese, S. M. Adil, G. Cofer, C. S. Perone, J. CohenAdad, S. P. Lad, and G. A. Johnson, Postmortem Diffusion MRI of the Entire Human Spinal Cord at Microscopic Resolution, NeuroImage: Clinical 18, 963 (2018).

[10] K. H. Maier-Hein, P. F. Neher, J.-C. Houde, M.-A. Côté, E. Garyfallidis, J. Zhong, and M. Chamberland, The Challenge of Mapping the Human Connectome Based on Diffusion Tractography, Nat. Commun. 8, 1349 (2017).

[11] V. J. Wedeen, D. L. Rosene, R. Wang, G. Dai, F. Mortazavi, P. Hagmann, J. H. Kaas, and W.-Y. I. Tseng, The Geometric Structure of the Brain Fiber Pathways, Science 335, 1628 (2012).

[12] V. J. Wedeen, D. L. Rosene, R. Wang, G. Dai, F. Mortazavi, P. Hagmann, J. H. Kaas, and W.-Y. I. Tseng, Response to Comment on "The Geometric Structure of the Brain Fiber Pathways”, Science 337, 1605 (2012).

[13] M. Catani, I. Bodi, and F. Dell'Acqua, Comment on "The Geometric Structure of the Brain Fiber Pathways", Science 337, 1605 (2012).

[14] K. Zilles, The Cortex of the Rat-A Stereotaxic Atlas (Springer, Berlin, 1985).

[15] K. Amunts, C. Lepage, L. Borgeat, H. Mohlberg, T. Dickscheid, M.-É. Rousseau, S. Bludau, P.-L. Bazin, L. B. Lewis, A.-M. Oros-Peusquens, N. J. Shah, T. Lippert, K. Zilles, and A.C. Evans, Bigbrain: An UltrahighResolution 3D Human Brain Model, Science 340, 1472 (2013).

[16] V. Carriel, A. Campos, M. Alaminos, S. Raimondo, and S. Geuna, Staining Methods for Normal and Regenerative Myelin in the Nervous System, in Histochemistry of Single Molecules: Methods and Protocols, edited by C. Pellicciari and M. Biggiogera (Springer, New York, 2017), pp. 207-218.

[17] J. Lanciego, F. Wouterlood, E. Erro, J. Arribas, N. Gonzalo, X. Urra, S. Cervantes, and J. Giménez-Amaya, Complex Brain Circuits Studied via Simultaneous and Permanent Detection of Three Transported Neuroanatomical Tracers in the Same Histological Section, J. Neurosci. Methods 103, 127 (2000).

[18] R. S. Cajal, Textura del Sistema Nervioso del Hombre y de los Vertebrados (Moya, Madrid, 1904).

[19] R. Nieuwenhuys, The Myeloarchitectonic Studies on the Human Cerebral Cortex of the Vogt-Vogt school, and 
Their Significance for the Interpretation of Functional Neuroimaging Data, Brain Struct. Funct. 218, 303 (2012).

[20] P. Osten and T. W. Margie, Mapping Brain Circuitry with a Light Microscope, Nat. Methods 10, 515 (2013).

[21] J. Men, Y. Huang, J. Solanki, X. Zeng, A. Alex, J. Jerwick, Z. Zhang, R. E. Tanzi, A. Li, and C. Zhou, Optical Coherence Tomography for Brain Imaging and Developmental Biology, IEEE J. Sel. Top. Quantum Electron. 22, 120 (2016).

[22] C. Magnain, J. C. Augustinack, E. Konukoglu, M. P. Frosch, S. Sakadžić, A. Varjabedian, N. Garcia, V. J. Wedeen, D. A. Boas, and B. Fischl, Optical Coherence Tomography Visualizes Neurons in Human Entorhinal Cortex, Neurophotonics 2, 015004 (2015).

[23] J. B. Arous, J. Binding, J. F. Léger, M. Casado, P. Topilko, S. Gigan, A. C. Boccara, and L. Bourdieu, Single Myelin Fiber Imaging in Living Rodents without Labeling by Deep Optical Coherence Microscopy, J. Biomed. Opt. 16, 116012 (2011).

[24] A. Li, H. Gong, B. Zhang, Q. Wang, C. Yan, J. Wu, Q. Liu, S. Zeng, and Q. Luo, Micro-optical Sectioning Tomography to Obtain a High-Resolution Atlas of the Mouse Brain, Science 330, 1404 (2010).

[25] E. A. Susaki, K. Tainaka, D. Perrin, F. Kishino, T. Tawara, T. M. Watanabe, C. Yokoyama, H. Onoe, M. Eguchi, S. Yamaguchi, T. Abe, H. Kiyonari, Y. Shimizu, A. Miyawaki, H. Yokota, and H. R. Ueda, Whole-Brain Imaging with Single-Cell Resolution Using Chemical Cocktails and Computational Analysis, Cell 157, 726 (2014).

[26] I. Costantini, J.-P. Ghobril, A. P. D. Giovanna, A. L. A. Mascaro, L. Silvestri, M. C. Müllenbroich, L. Onofri, V. Conti, F. Vanzi, L. Sacconi, R. Guerrini, H. Makram, G. Iannello, and F. S. Pavone, A Versatile Clearing Agent for Multi-modal Brain Imaging, Sci. Rep. 5, 9808 (2015).

[27] J. Mertz and J. Kim, Scanning Light-Sheet Microscopy in the Whole Mouse Brain with HiLo Background Rejection, J. Biomed. Opt. 15, 016027 (2010).

[28] M. Stefaniuk, E. J. Gualda, M. Pawlowska, D. Legutko, P. Matryba, P. Koza, W. Konopka, D. Owczarek, M. Wawrzyniak, P. Loza-Alvarez, and L. Kaczmarek, LightSheet Microscopy Imaging of a Whole Cleared Rat Brain with Thyl-GFP Transgene, Sci. Rep. 6, 28209 (2016).

[29] L. Silvestri, A. L. A. Mascaro, I. Costantini, L. Sacconi, and F.S. Pavone, Correlative Two-Photon and Light Sheet Microscopy, Methods 66, 268 (2014).

[30] K. Wang, N. G. Horton, and C. Xu, Going Deep: Brain Imaging with Multiphoton Microscopy, Opt. Photonics News 24, 32 (2013).

[31] W. Zong, R. Wu, M. Li, Y. Hu, Y. Li, J. Li, H. Rong, H. Wu, Y. Xu, Y. Lu, H. Jia, M. Fan, Z. Zhou, Y. Zhang, A. Wang, L. Chen, and H. Cheng, Fast High-Resolution Miniature Two-Photon Microscopy for Brain Imaging in Freely Behaving Mice, Nat. Methods 14, 713 (2017).

[32] S. P. Amato, F. Pan, J. Schwartz, and T. M. Ragan, Whole Brain Imaging with Serial Two-Photon Tomography, Front. Neuroanat. 10, 31 (2016).

[33] R. Kawakami, K. Sawada, A. Sato, T. Hibi, Y. Kozawa, S. Sato, H. Yokoyama, and T. Nemoto, Visualizing Hippocampal Neurons with In Vivo Two-Photon Microscopy
Using a $1030 \mathrm{~nm}$ Picosecond Pulse Laser, Sci. Rep. 3, 1014 (2013).

[34] M. Axer, K. Amunts, D. Grässel, C. Palm, J. Dammers, H. Axer, U. Pietrzyk, and K. Zilles, A Novel Approach to the Human Connectome: Ultra-high Resolution Mapping of Fiber Tracts in the Brain, NeuroImage 54, 1091 (2011).

[35] M. Axer, D. Grässel, M. Kleiner, J. Dammers, T. Dickscheid, J. Reckfort, T. Hütz, B. Eiben, U. Pietrzyk, K. Zilles, and K. Amunts, High-Resolution Fiber Tract Reconstruction in the Human Brain by Means of ThreeDimensional Polarized Light Imaging, Front. Neuroinform. 5, 34 (2011).

[36] M. Menzel, K. Michielsen, H. De Raedt, J. Reckfort, K. Amunts, and M. Axer, A Jones Matrix Formalism for Simulating Three-Dimensional Polarized Light Imaging of Brain Tissue, J. R. Soc. Interface 12, 20150734 (2015).

[37] F. O. Schmitt and R. S. Bear, The Ultrastructure of the Nerve Axon Sheath, Biol. Rev. Camb. Philos. Soc. 14, 27 (1939).

[38] M. Koike-Tani, T. Tani, S. B. Mehta, A. Verma, and R. Oldenbourg, Polarized Light Microscopy in Reproductive and Developmental Biology, Mol. Reprod. Dev. 82, 548 (2015).

[39] R. E. Martenson, Myelin: Biology and Chemistry (CRC Press, Boca Raton, 1992).

[40] S. Caspers and M. Axer, Decoding the Microstructural Correlate of Diffusion MRI, NMR Biomed. 32, e3779 (2019).

[41] M. Zeineh, N. Palomero-Gallagher, M. Axer, D. Gräßel, M. Goubran, A. Wree, R. Woods, K. Amunts, and K. Zilles, Direct Visualization and Mapping of the Spatial Course of Fiber Tracts at Microscopic Resolution in the Human Hippocampus, Cereb. Cortex 27, 1779 (2016).

[42] H. Wiese, D. Grässel, U. Pietrzyk, K. Amunts, and M. Axer, Polarized Light Imaging of the Human Brain: A New Approach to the Data Analysis of Tilted Sections, in SPIE Proceedings, Polarization: Measurement, Analysis, and Remote Sensing XI, Vol. 9099, edited by D. B. Chenault and D. H. Goldstein (SPIE, Bellingham, WA, 2014).

[43] D. Schmitz, K. Amunts, T. Lippert, and M. Axer, A Least Squares Approach for the Reconstruction of Nerve Fiber Orientations from Tiltable Specimen Experiments in 3DPLI, in Proceedings of the 2018 IEEE 15th International Symposium on Biomedical Imaging (ISBI 2018), Washington, DC (IEEE, New York, 2018), pp. 132-135.

[44] I. Costantini, M. Menzel, L. Silvestri, N. Schubert, M. Axer, K. Amunts, and F.S. Pavone, Polarized Light Imaging and Two-Photon Fluorescence Microscopy Correlative Approach for $3 D$ Reconstruction of the Orientation of Myelinated Fibers, Optics in the Life Sciences Congress, OSA Technical Digest, (Optical Society of America, 2017) paper BrW4B.5, https://doi.org/10.1364/BRAIN.2017. BrW4B.5.

[45] A. Taflove and S. C. Hagness, Computational Electrodynamics: The Finite-Difference Time-Domain Method (Artech House, Boston, 2000).

[46] M. Menzel, M. Axer, H. De Raedt, and K. Michielsen, Finite-Difference Time-Domain Simulation for ThreeDimensional Polarized Light Imaging, in Brain-Inspired Computing. BrainComp 2015, Lecture Notes in Computer 
Science Vol. 10087, edited by K. Amunts, L. Grandinetti, T. Lippert, and N. Petkov (Springer International, Cham, 2016), Chap. 6, pp. 73-85.

[47] B. D. Wilts, K. Michielsen, H. De Raedt, and D. G. Stavenga, Sparkling Feather Reflections of a Bird-ofParadise Explained by Finite-Difference Time-Domain Modeling, Proc. Natl. Acad. Sci. U.S.A. 111, 4363 (2014).

[48] H. De Raedt, K. Michielsen, and K. Hess, Analysis of Multipath Interference in Three-Slit Experiments, Phys. Rev. A 85, 012101 (2012).

[49] B. D. Wilts, K. Michielsen, J. Kuipers, H. De Raedt, and D. G. Stavenga, Brilliant Camouflage: Photonic Crystals in the Diamond Weevil, Entimus imperialis, Proc. R. Soc. B 279, 2524 (2012).

[50] H.-J. Schwarzmaier, A. Yaroslavsky, I. Yaroslavsky, T. Goldbach, T. Kahn, F. Ulrich, P. C. Schulze, and R. Schober, Optical Properties of Native and Coagulated Human Brain Structures, Proc. SPIE Int. Soc. Opt. Eng. 2970, 492 (1997).

[51] A. N. Yaroslavsky, P. C. Schulze, I. V. Yaroslavsky, R. Schober, F. Ulrich, and H.-J. Schwarzmaier, Optical Properties of Selected Native and Coagulated Human Brain Tissues In Vitro in the Visible and Near Infrared Spectral Range, Phys. Med. Biol. 47, 2059 (2002).

[52] R. P. Woods, S. C. Fears, M. J. Jorgensen, L. A. Fairbanks, A. W. Toga, and N. B. Freimer, A Web-Based Brain Atlas of the Vervet Monkey, Chlorocebus aethiops, NeuroImage 54, 1872 (2011).

[53] http://www.loni.usc.edu/Research/Atlases/Data/vervet/ vervetatlas/vervetatlas.html, University of Southern California, Laboratory of Neuro Imaging, 2018.

[54] K. Zilles, N. Palomero-Gallagher, D. Gräßel, P. Schlömer, M. Cremer, R. Woods, K. Amunts, and M. Axer, HighResolution Fiber and Fiber Tract Imaging Using Polarized Light Microscopy in the Human, Monkey, Rat, and Mouse Brain, in Axons and Brain Architecture, edited by K. S. Rockland (Elsevier, San Diego, 2015), Chap. 18, pp. 369-389.

[55] See Supplemental Material at http://link.aps.org/ supplemental/10.1103/PhysRevX.10.021002 for additional figures.

[56] M. Dohmen, M. Menzel, H. Wiese, J. Reckfort, F. Hanke, U. Pietrzyk, K. Zilles, K. Amunts, and M. Axer, Understanding Fiber Mixture by Simulation in 3D Polarized Light Imaging, NeuroImage 111, 464 (2015).

[57] U. Bürgel, K. Amunts, L. Hoemke, H. Mohlberg, J. M. Gilsbach, and K. Zilles, White Matter Fiber Tracts of the Human Brain: Three-Dimensional Mapping at Microscopic Resolution, Topography and Intersubject Variability, NeuroImage 29, 1092 (2006).

[58] M. T. de Schotten, D. H. ffytche, A. Bizzi, F. Dell'Acqua, M. Allin, M. Walshe, R. Murray, S. C. Williams, D. G. Murphy, and M. Catani, Atlasing Location, Asymmetry and Inter-subject Variability of White Matter Tracts in the Human Brain with MR Diffusion Tractography, NeuroImage 54, 49 (2011).

[59] Advances in FDTD Computational ElectrodynamicsPhotonics and Nanotechnology, edited by A. Taflove (Artech House, Boston, 2013).

[60] J. T. Azpiroz, G. W. Burr, A. E. Rosenbluth, and M. Hibbs, Massively-Parallel FDTD Simulations to Address Mask
Electromagnetic Effects in Hyper-NA Immersion Lithography, Proc. SPIE 6924, 69240Y (2008).

[61] A. Erdmann, P. Evanschitzky, G. Citarella, T. Fühner, and P. D. Bisschop, Rigorous Mask Modeling Using Waveguide and FDTD Methods: An Assessment for Typical Hyper-NA Imaging Problems, Proc. SPIE 6283, 628319 (2006).

[62] J. M. McMahon, S. K. Gray, and G. C. Schatz, Calculating Nonlocal Optical Properties of Structures with Arbitrary Shape, Phys. Rev. B 82, 035423 (2010).

[63] A. S. Grimault, A. Vial, and M. L. de la Chapelle, Modeling of Regular Gold Nanostructures Arrays for SERS Applications Using a 3D FDTD Method, Appl. Phys. B 84, 111 (2006).

[64] M. Irannejad, M. Yavuz, and B. Cui, Finite Difference Time Domain Study of Light Transmission through Multihole Nanostructures in Metallic Film, Photon. Res. 1, 154 (2013).

[65] R. Drezek, A. Dunn, and R. Richards-Kortum, A Pulsed Finite-Difference Time-Domain (FDTD) Method for Calculating Light Scattering from Biological Cells over Broad Wavelength Ranges, Opt. Express 6, 147 (2000).

[66] P. Kosmas, C. Rappaport, and E. Bishop, Modeling with the FDTD Method for Microwave Breast Cancer Detection, IEEE Trans. Microwave Theory Tech. 52, 1890 (2004).

[67] M. Menzel, Finite-Difference Time-Domain Simulations Assisting to Reconstruct the Brain's Nerve Fiber Architecture by 3D Polarized Light Imaging, Schriften des Forschungszentrums Jülich, Reihe Schlüsseltechnologien, Vol. 188 (Forschungszentrum Jülich GmbH, Jülich, 2018).

[68] K. Michielsen, H. de Raedt, and D. G. Stavenga, Reflectivity of the Gyroid Biophotonic Crystals in the Ventral Wing Scales of the Green Hairstreak Butterfly, Callophrys rubi, J. R. Soc. Interface 7, 765 (2010).

[69] N.-J. Jan, J. L. Grimm, H. Tran, K. L. Lathrop, G. Wollstein, R. A. Bilonick, H. Ishikawa, L. Kagemann, J. S. Schuman, and I. A. Sigal, Polarization Microscopy for Characterizing Fiber Orientation of Ocular Tissues, Biomed. Opt. Express 6, 4705 (2015).

[70] N.-J. Jan, K. Lathrop, and I. A. Sigal, Collagen Architecture of the Posterior Pole: High-Resolution Wide Field of View Visualization and Analysis Using Polarized Light Microscopy, Invest. Ophthalmol. Visual Sci. 58, 735 (2017).

[71] M. Menzel, J. Reckfort, D. Weigand, H. Köse, K. Amunts, and M. Axer, Diattenuation of Brain Tissue and Its Impact on 3D Polarized Light Imaging, Biomed. Opt. Express 8, 3163 (2017).

[72] M. Menzel, M. Axer, K. Amunts, H. D. Raedt, and K. Michielsen, Diattenuation Imaging Reveals Different Brain Tissue Properties, Sci. Rep. 9, 1939 (2019).

[73] M. M. Woolfson, The Development of Structural X-Ray Crystallography, Phys. Scr. 93, 032501 (2018).

[74] D. Krause and P. Thörnig, JURECA: General-Purpose Supercomputer at Jülich Supercomputing Centre, J. LargeScale Res. Facil. 4, A132 (2016).

[75] M. Stephan and J. Docter, JUQUEEN: IBM Blue Gene/Q Supercomputer System at the Jülich Supercomputing Centre, Large-Scale Res. Fac. 1, A1 (2015).

[76] J. Reckfort, H. Wiese, U. Pietrzyk, K. Zilles, K. Amunts, and M. Axer, A Multiscale Approach for the Reconstruction 
of the Fiber Architecture of the Human Brain Based on 3D-PLI, Front. Neuroanat. 9, 118 (2015).

[77] S. Klein, M. Staring, K. Murphy, M. A. Viergever, and J.P.W. Pluim, elastix: A Toolbox for Intensity-Based Medical Image Registration, IEEE Trans. Med. Imaging 29, 196 (2010).

[78] D. P. Shamonin, E. E. Bron, B. P. F. Lelieveldt, M. Smits, S. Klein, and M. Staring, Fast Parallel Image Registration on CPU and GPU for Diagnostic Classification of Alzheimer's Disease, Front. Neuroinform. 7, 50 (2013).

[79] B. B. Avants, C. L. Epstein, M. Grossman, and J. C. Gee, Symmetric Diffeomorphic Image Registration with CrossCorrelation: Evaluating Automated Labeling of Elderly and Neurodegenerative Brain, Med. Image Anal. 12, 26 (2008).

[80] B. B. Avants, N. J. Tustison, G. Song, P. A. Cook, A. Klein, and J.C. Gee, A Reproducible Evaluation of ANTs Similarity Metric Performance in Brain Image Registration, NeuroImage 54, 2033 (2011).

[81] National library of medicine insight segmentation and registration toolkit (ITK), https://itk.org/.

[82] D. Wagner and D. Schmalstieg, ARToolKitPlus for Pose Tracking on Mobile Devices, in Proceedings of the 12th Computer Vision Winter Workshop (2007), pp. 139-146, https://www.semanticscholar.org/paper/Artoolkitplus-forpose-tracking-on-mobile-devices-Wagner-Schmalstieg/ d411c076ba457d0c13c5ddc9b7f24246cf135a5b.

[83] M. Schober, P. Schlömer, M. Cremer, H. Mohlberg, A.-M. Huynh, N. Schubert, M. E. Kirlangic, K. Amunts, and M. Axer, Reference Volume Generation for Subsequent 3D Reconstruction of Histological Sections, in Bildverarbeitung für die Medizin 2015. Informatik aktuell, edited by $\mathrm{H}$. Handels, T. Deserno, H. P. Meinzer, and T. Tolxdorff (Springer Verlag, Berlin, 2015), pp. 143-148.

[84] G. Paxinos and C. Watson, The Rat Brain in Stereotaxic Coordinates, 6th ed. (Academic, New York, 2007).

[85] E. A. Papp, T. B. Leergard, E. Calabrese, G. A. Johnson, and J. G. Bjaalie, Waxholm Space Atlas of the Sprague Dawley Rat Brain, NeuroImage 97, 374 (2014).

[86] F. Matuschke, K. Ginsburger, C. Poupon, K. Amunts, and M. Axer, Dense Fiber Modeling for 3D-Polarized Light Imaging Simulations, arXiv:1901.10284.

[87] R. H. Quarles, W. B. Macklin, and P. Morell, Myelin Formation, Structure and Biochemistry, in Basic
Neurochemistry: Molecular, Cellular and Medical Aspects, 7th ed., edited by G. Siegel, R. W. Albers, S. Brady, and D. Price (Elsevier Academic Press, Burlington, MA, 2006), pp. $51-71$.

[88] C. Hildebrand, S. Remahl, H. Persson, and C. Bjartmar, Myelinated Nerve Fibers in the CNS, Prog. Neurobiol. 40, 319 (1993).

[89] D. W. Lee, X. Banquy, K. Kristiansen, Y. Kaufman, J. M. Boggs, and J. N. Israelachvili, Lipid Domains Control Myelin Basic Protein Adsorption and Membrane Interactions between Model Myelin Lipid Bilayers, Proc. Natl. Acad. Sci. U.S.A. 111, E768 (2014).

[90] H.-J. van Manen, P. Verkuijlen, P. Wittendorp, V. Subramaniam, T. K. van den Berg, D. Roos, and C. Otto, Refractive Index Sensing of Green Fluorescent Proteins in Living Cells Using Fluorescence Lifetime Imaging Microscopy, Biophys. Rev. Lett. 94, L67 (2008).

[91] F. A. Duck, Physical Properties of Tissue: A Comprehensive Reference Book (Academic, San Diego, 1990).

[92] P. Morell, R. H. Quarles, and W. T. Norton, Formation, Structure, and Biochemistry of Myelin, in Basic Neurochemistry-Molecular, Cellular, and Medical Aspects, 4th ed., edited by G. J. Siegel (Raven Press, New York, 1989), pp. 109-136.

[93] K. S. Yee, Numerical Solution of Initial Boundary Value Problems Involving Maxwell's Equations in Isotropic Media, IEEE Trans. Antennas Propag. 14, 302 (1966).

[94] H. De Raedt, Advances in Unconditionally Stable Techniques, in Computational Electrodynamics: The FiniteDifference Time-Domain Method (Artech House, Boston, 2005).

[95] R. C. Jones, A New Calculus for the Treatment of Optical Systems, J. Opt. Soc. Am. 31, 488 (1941).

[96] E. Collett, Field Guide to Polarization (SPIE-The International Society for Optical Engineering, Bellingham, WA, 2005).

[97] T. E. Oliphant, Python for Scientific Computing, Comput. Sci. Eng. 9, 10 (2007).

[98] S. van der Walt, S. C. Colbert, and G. Varoquaux, The NumPy Array: A Structure for Efficient Numerical Computation, Comput. Sci. Eng. 13, 22 (2011).

[99] J. W. Cooley and J. W. Tukey, An Algorithm for the Machine Calculation of Complex Fourier Series, Math. Comput. 19, 297 (1965). 